TITLE:

\title{
Nonlinear evolution of cosmic magnetic fields and cosmic microwave background anisotropies
}

\section{$\operatorname{AUTHOR(S):~}$}

Tashiro, H; Sugiyama, N; Banerjee, R

\section{CITATION:}

Tashiro, H ...[et al]. Nonlinear evolution of cosmic magnetic fields and cosmic microwave background anisotropies. PHYSICAL REVIEW D 2006, 73(2): 023002.

ISSUE DATE:

2006-01

URL:

http://hdl.handle.net/2433/49990

RIGHT:

Copyright 2006 American Physical Society 
PHYSICAL REVIEW D 73, 023002 (2006)

\title{
Nonlinear evolution of cosmic magnetic fields and cosmic microwave background anisotropies
}

\author{
Hiroyuki Tashiro* \\ Department of Physics, Kyoto University, Kyoto 606-8502, Japan \\ Naoshi Sugiyama \\ Division of Theoretical Astronomy, National Astronomical Observatory, Japan, Mitaka, Tokyo 181-8588 Japan \\ Robi Banerjee \\ Department of Physics and Astronomy, McMaster University, Hamilton, Ontario L8S 4M1, Canada
}

(Received 7 September 2005; published 9 January 2006; corrected 14 June 2006)

\begin{abstract}
In this work we investigate the effects of primordial magnetic fields on cosmic microwave background anisotropies (CMB). Based on cosmological magneto-hydro dynamic (MHD) simulations [R. Banerjee and K. Jedamzik, Phys. Rev. D 70, 123003 (2004).] we calculate the CMB anisotropy spectra and polarization induced by fluid fluctuations (Alfvén modes) generated by primordial magnetic fields. The strongest effect on the CMB spectra comes from the transition epoch from a turbulent regime to a viscous regime. The balance between magnetic and kinetic energy until the onset of the viscous regime provides a one to one relation between the comoving coherence length $L$ and the comoving magnetic field strength $B$, such as $L \sim 30\left(B / 10^{-9} \mathrm{Gauss}\right)^{3} \mathrm{pc}$. The resulting CMB temperature and polarization anisotropies for the initial power law index of the magnetic fields $n>3 / 2$ are somewhat different from the ones previously obtained by using linear perturbation theory. In particular, differences can appear on intermediate scales $l<2000$ and small scales $l>20000$. On scales $l<2000$ the CMB anisotropy and polarization spectra are flat in the case of our nonlinear calculations whereas the spectra have a blue index calculated with linear perturbation theory if we assume the velocity fields of baryons induced by the magnetic fields achieved Alfvén velocity due to the turbulent motions on large scales in the early universe. Our calculation gives a constraint on the magnetic field strength in the intermediate scale of CMB observations. Upper limits are set by WMAP and BOOMERANG results for comoving magnetic field strength of $B<$ 28 nGauss with a comoving coherence length of $L>0.7 \mathrm{Mpc}$ for the most extreme case, or $B<$ 30 nGauss and $L>0.8 \mathrm{Mpc}$ for the most conservative case. We may also expect higher signals on large scales of the polarization spectra compared to linear calculations. The signal may even exceed the B-mode polarization from gravitational lensing depending on the strength of the primordial magnetic fields. On very small scales, the diffusion damping scale of nonlinear calculations turns out to be much smaller than the one of linear calculations if the comoving magnetic field strength $B>16$ nGauss. If the magnetic field strength is smaller, the diffusion scales become smaller too. Therefore we expect to have both, temperature and polarization anisotropies, even beyond $l>10000$ regardless of the strength of the magnetic fields. The peak values of the temperature anisotropy and the B-mode polarization spectra are approximately $40 \mu \mathrm{K}$ and a few $\mu \mathrm{K}$, respectively.
\end{abstract}

DOI: 10.1103/PhysRevD.73.023002

PACS numbers: 98.70.Vc, 98.62.En

\section{INTRODUCTION}

Magnetic fields have been observed in many galaxies and galaxy clusters $[1,2]$. Observations have revealed that these magnetic fields typically have a few $\mu$ Gauss strengths and relatively large coherent scales, i.e., a few tens of kpc for clusters of galaxies and a few kpc for galaxies. It is one of the great challenges for modern astronomy to understand the origin of these magnetic fields.

Perhaps the most conventional scenario of generating such magnetic fields is as follows. First, small seeds of the magnetic fields are produced due to the Biermann battery mechanism. Although the resultant magnetic fields are very weak, those are amplified by the dynamo process [3] (for a comprehensive review see [4]). Eventually these

*Electronic address: htashiro@tap.scphys.kyoto-u.ac.jp magnetic fields are spread by Supernova winds or active galactic nuclei jets into intergalactic medium.

However, the fact that magnetic fields with very large coherent scales are observed in galaxy clusters or high redshift galaxies with a strength of a few $\mu$ Gauss casts some questions on this standard dynamo scenario [1,5]: how can these coherent magnetic fields be spread into intercluster medium, and is it possible for the dynamo amplification to take place fast enough in high redshift galaxies? An alternative possibility to the dynamo scenario is the generation of magnetic fields in the early universe. There are in fact many previous works which suggest the generation of the magnetic fields in the early universe, e.g. during an inflation or at cosmological phase transitions (QCD or electroweak phase transition). For a detailed review, see [6].

If magnetic fields are generated in the early universe, we need to understand their evolution in the expanding hot 
universe to obtain their field strength and structure at present. Several studies of evolution of magnetic fields have been performed by employing the linearized equations of magneto-hydro dynamics (MHD) [7,8]. These studies found that the growth of magnetic fields in the early universe is rather complicated such that the cosmological viscosity plays an important role [7]. Magnetic field energy is dissipated by the viscosity due to neutrinos and photons, equivalent to Silk damping [9] for density fluctuations of baryon-photon fluid. However, the damping efficiency is different for different MHD modes. The Alfvén and the slow modes are damped less than the fast modes so that the Alfvén and the slow modes survive at small scales where the dissipation is effective. Meanwhile, it is also expected that the nonlinear effects contribute to the evolution of magnetic fields since equipartition between the magnetic field and the fluid will be established and the magnetic fields will cascade from large scales to small scales. Nonlinear effects on Alfvén modes in the presence of viscosity is investigated analytically for particular configurations [8] and only a little damping of Alfvén modes is found in these situations.

Recently, Banerjee and Jedamzik [10,11] studied the evolution of Alfvén modes using MHD simulation in the expanding universe including dissipation due to diffusion and neutrino/photon-drag due to free-streaming neutral particles. The evolution of the primordial magnetic fields is solely determined by the kinetic Reynolds number, which is defined by $R \equiv v^{2} / L f$ where $v$ is the fluid velocity, $L$ is the length scale and $f$ is the fluid dissipation (which is discussed in more detail in the following section), can be divided into three different regimes: turbulent regime $(R \gg 1)$, viscous (diffusion) regime and (viscous) free-streaming regime $(R<1)$. Furthermore, analytic expressions for the growth of the magnetic coherence length due to small wavelength damping and helicity conservation were found. Under optimistic assumptions it is possible for magnetic fields produced during a QCD phase transition with an initial coherence length of $1 \mathrm{pc}$ to have attained a kpc coherence length at present.

The existence of the primordial magnetic fields leaves traces on various cosmological phenomena. Investigating the cosmological effects of the magnetic fields in the early universe, i.e., the effects on Big Bang nucleosynthesis (BBN), structure formation and cosmological microwave background (CMB) anisotropies, we can constrain the strength and the structure of primordial magnetic fields.

Among them, the traces of primordial magnetic fields on $\mathrm{CMB}$ anisotropies and polarization are of particular interest since they provide information of not only the magnetic field energy but also the coherence length. On the contrary, BBN gives only a limit on the magnetic field energy density ( $B \lesssim 7 \times 10^{-5}$ Gauss at present) [12].

The coherence length is an especially important observational quantity if one wishes to understand the origin of cosmic magnetic fields because the length scale strongly correlates with the production mechanism. The primordial magnetic fields produced by a causal mechanism during, for example, a phase transition are limited by the length scale which corresponds to the horizon scale at the epoch of the magnetic filed generation. In contrast, the magnetic fields produced in the inflation epoch are expected to have the scale-invariant spectrum such that the amplitudes of the magnetic fields at the horizon crossing are the same over the all scales.

There have been already several works to set constraints on the primordial magnetic fields by using current $\mathrm{CMB}$ data. But so far, non of these considered the full nonlinear evolution of Alfvén modes. Barrow et al. [13] set a limit on the homogeneous (coherent) magnetic field by using COBE data. Such a homogeneous magnetic field produces large scale anisotropic pressures and these pressures require an anisotropic gravitational field to support them. This anisotropic gravitational field should generate anisotropies in CMB temperature. They obtain a limit of $B \lesssim 10^{-9}$ Gauss [13] at present.

Primordial magnetic fields with a coherence length comparable to the horizon size at the last scattering surface (LSS) affect the sound speed of the baryon-photon fluid and change their acoustic oscillations. This effect might be observed as the modification of the acoustic peaks in the CMB angular power spectrum. Adams et al. [14] found that this modification should be detectable by WMAP and PLANCK if the today's magnetic fields are $B>10^{-8}$ Gauss with the coherence length larger than the horizon at recombination.

A distortion of the CMB energy spectrum from the black body shape also gives a constraint on primordial magnetic fields. The magnetic fields with a small coherent scale are dissipated due to viscosity of the baryon-photon fluid and this dissipation energy distorts the black body spectrum of CMB [15]. The spectrum distortion is described by either a chemical potential $\mu$ or a Compton $y$-parameter. If the dissipation process takes place in the very early universe when thermal equilibrium is maintained, it does not affect the $\mathrm{CMB}$ spectrum. As the temperature of the universe goes down, however, thermal equilibrium can no longer be maintained. Instead, kinetic equilibrium is preserved since only the Compton scattering process which conserves photon number is effective. If dissipation occurs during this stage, we expect to have $\mu$ distortion. As the temperature drops more, eventually even kinetic equilibrium can no longer be maintained and we expect to have $y$ distortion due to the dissipation. COBE-FIRAS gives the CMB chemical potential constraint of $\mu \leqq 9 \times 10^{-5}$. This limit corresponds to the magnetic field constraint of $B \leqq 3 \times 10^{-8}$ Gauss at present on a comoving scale of $400 \mathrm{pc}$ at redshift $z \gtrsim 2 \times 10^{6}$ [15]. On the other hand, the constraint by the Compton $y$-parameter, which is $y \lesssim$ $1.5 \times 10^{-5}$, leads to the constraint on the magnetic fields of $B \leqslant 3 \times 10^{-8}$ Gauss on $600 \mathrm{kpc}$. 
Another important constraint can be given from small scale CMB temperature anisotropies and polarization. Magnetic fields induce peculiar velocities in photonbaryon fluids driven by Alfvén modes. These peculiar velocities generate temperature anisotropies and polarization in CMB. Observations by WMAP already constrain these anisotropies induced by Alfvén modes to be smaller than the primary anisotropies due to acoustic oscillations of the photon-baryon fluid. Therefore, one might conclude that there is no chance to measure magnetic field sourced temperature fluctuations. However this is not the case for small scale anisotropies. Primary CMB anisotropies and polarization at the recombination epoch are expected to have a lack of small scale power due to the diffusion damping of the photon-baryon fluid (Silk damping). On the other hand, calculations of linear evolution of Alfvén modes show less damping [16-19]. These Alfvén modes generate $\mathrm{CMB}$ temperature anisotropies and polarization. Therefore we can likely expect to have dominant contribution on small scale CMB anisotropies from the magnetic fields if they exist. Using the WMAP result and taking into account both scalar and Alfvén modes, one can constrain primordial magnetic fields to be $B \leqq 3.9 \times 10^{-9}$ Gauss on $1 \mathrm{Mpc}$ at present by using the Markov Chain Monte Carlo method [19].

For the same reason, polarization induced by Alfvén modes from primordial magnetic fields may dominate on small scales [20]. It is known that the polarization can be decomposed into two modes, i.e., the E-mode polarization (gradient component) and the B-mode polarization (rotation component) [21]. Observationally they are distinguishable. It is very interesting that Alfvén modes only produce B-mode polarization since its perturbations are of vector type. On the contrary, scalar type perturbations, which form the large scale structure of the universe, only produce E-mode polarization. It has been known that the gravitational lensing effect on the primary E-mode polarization caused by structure of the universe produces Bmode polarization on intermediate scales (a few tens of arc minute angular size). However, polarization of the magnetic field origin can be dominant as a B-mode on small sales.

A few authors have investigated the generation of Bmode polarization either by an analytic treatment with employing the tight coupling approximation [22] or by direct numerical calculations [17,18]. For the evolution of Alfvén mode perturbations, however, they all apply linear analysis. In this paper, we study the evolution of Alfvén modes using recent MHD simulation by Banerjee and Jedamzik $[10,11]$, which allows us to incorporate the fully nonlinear and self consistent spectra of the Alfvén modes to calculate resulting CMB temperature anisotropy and polarization spectra.

This paper is organized as follows. In Sec. II, we summarize the evolution of Alfvén modes based on the nu- merical MHD simulation of Banerjee and Jedamzik. We separate the evolution into three regimes, i.e., turbulent, viscous and free-streaming regimes. In each regime, the evolution of velocity fields is carefully investigated. In Sec. III, the power spectrum of the velocity fields is calculated. Using this power spectrum, in Sec. IV, we compute CMB temperature anisotropy and polarization spectra. Sec. V is devoted to discussion, in which we compare our results to those obtained with linear perturbation theory. We give our conclusions in Sec. VI. Throughout the paper, we take WMAP values for the cosmological parameters, i.e., $h=0.71\left(H_{0}=h \times 100 \mathrm{Km} / \mathrm{s} \cdot \mathrm{Mpc}\right)$, $T_{0}=2.725 \mathrm{~K}, \Omega_{\mathrm{b}} h^{2}=0.0224$ and $\Omega_{\mathrm{M}} h^{2}=0.135$ [23].

\section{EVOLUTION OF ALFVÉN MODES}

Our final goal is to investigate the effect of the primordial magnetic fields on the CMB temperature anisotropies and polarization. Magnetic stresses produce vortical modes, such as the "Alfvén mode" and "slow magnetosonic mode" in the ionized fluids. As both are very similar we will refer to them as Alfvén modes, henceforth. These Alfvén modes generate additional temperature anisotropies and polarization by Doppler shift. Therefore, we need to know the evolution of Alfvén modes first. Unlike previous works which employed a linear perturbation approximation [16,17,22], Banerjee and Jedamzik [10,11] recently investigated the nonlinear evolution of magnetic fields and Alfvén modes using numerical MHD simulation. These simulations cover the three different damping regimes appearing in the early universe: turbulence, viscous, and free-streaming. Based on their work, we summarize the evolution of the velocity fluctuations (Alfvén modes) of the ionized fluid in this section.

The growth of Alfvén modes is affected by the interaction with photons and/or neutrinos in the early universe. Here, we consider the interaction with photons only as neutrinos are already decoupled from the cosmic evolution at the epoch of LSS.

The MHD equations in the expanding universe including diffusion due to the photon background are given by [10]

$$
\begin{aligned}
\frac{\partial \mathbf{v}}{\partial t}+\frac{1}{a}(\mathbf{v} \cdot \nabla) \mathbf{v}+ & \left(1-3 c_{\mathrm{st}}^{2}\right) H \mathbf{v} \\
+ & \frac{1}{a}\left(\frac{\mathbf{B} \times(\nabla \times \mathbf{B})}{4 \pi\left(\rho_{\mathrm{t}}+p_{\mathrm{t}}\right)}\right)=\mathbf{f}, \\
\frac{\partial \mathbf{B}}{\partial t}+2 H \mathbf{B} & =\frac{1}{a} \nabla \times(\mathbf{v} \times \mathbf{B}),
\end{aligned}
$$

where $\rho_{\mathrm{b}}$ is the baryon density, $\rho_{\mathrm{t}}, p_{\mathrm{t}}$ and $c_{\mathrm{st}}$ denote the total density, pressure and sound velocity of photons and baryons, $H$ is the Hubble parameter and $\mathbf{f}$ is the dissipation term. The velocity $\mathbf{v}$ of photon-baryon fluids here is the Alfvén mode, and as such is incompressible so that $\mathbf{v}$ satisfies $\nabla \cdot \mathbf{v}=0$. The dissipation term, $\mathbf{f}$, is written as 


$$
\mathbf{f}=a^{-2} \nu(\rho+p)^{-1}\left(\nabla^{2} \mathbf{v}\right),
$$

where the shear viscosity $\nu$ is given by [24]

$$
\nu=\frac{4}{15} \rho_{\gamma} L_{\mathrm{mfp}} .
$$

Here $\rho_{\gamma}$ is the radiation energy density and $L_{\mathrm{mfp}}$ is the photon mean free path, $L_{\mathrm{mfp}}=\left(\sigma_{\mathrm{T}} n_{e}\right)^{-1}$ where $\sigma_{\mathrm{T}}$ is the Thomson cross section and $n_{e}$ is the free electron density.

By using $\nabla \cdot \mathbf{B}=0$, Eq. (1) is rewritten as

$$
\frac{\partial \mathbf{v}}{\partial t}+\frac{1}{a}(\mathbf{v} \cdot \nabla) \mathbf{v}+\left(1-3 c_{\mathrm{st}}^{2}\right) H \mathbf{v}-\frac{1}{a}\left(\mathbf{v}_{\mathrm{A}} \cdot \nabla\right) \mathbf{v}_{\mathrm{A}}=\mathbf{f},
$$

where $\mathbf{v}_{\mathrm{A}}$ is the Alfvén velocity, $\mathbf{v}_{\mathrm{A}} \equiv \mathbf{B} / \sqrt{4 \pi\left(\rho_{\mathrm{t}}+p_{\mathrm{t}}\right)}$.

The dissipation term, $\mathbf{f}$ which assumes multiple scattering between photons and baryons is valid only on scales larger than $L_{\mathrm{mfp}}$. Since $L_{\mathrm{mfp}}$ evolves rapidly as $a^{3}$, it soon exceeds the wave length of a particular mode we consider. In other word, the comoving wave number of the velocity, $k$, becomes $a / k<L_{\mathrm{mfp}}$. Subsequently, we need to treat baryons (ionized fluids) and photons separately.

Once the diffusive description becomes invalid, Alfvén modes will be damped due to free-streaming background photons. Note that the damping time on the integral scale in the viscous regime is longer than the Hubble time and dynamic evolution of the magnetic field is "stalled". In the free-streaming regime, the dissipation process can be described as radiation drag whose force is proportional to the fluid velocity $\mathbf{v}$. The coefficient $\alpha$ of the drag force is given by [25]

$$
\alpha=\frac{4}{3} \frac{\rho_{\gamma}}{\rho_{\mathrm{b}}} L_{\mathrm{mfp}}^{-1}
$$

Therefore, the Euler equation for the baryon fluid with free-streaming photons is given by

$$
\frac{\partial \mathbf{v}}{\partial t}+\frac{1}{a}(\mathbf{v} \cdot \nabla) \mathbf{v}+H \mathbf{v}-\frac{1}{a}\left(\frac{\rho_{\mathrm{t}}+p_{\mathrm{t}}}{\rho_{\mathrm{b}}}\right)\left(\mathbf{v}_{\mathrm{A}} \cdot \nabla\right) \mathbf{v}_{\mathrm{A}}=-\alpha \mathbf{v}
$$

Let us now consider a characteristic scale on which most of the magnetic energy exists. This characteristic scale corresponds to the peak of the magnetic field power spectrum and can be defined by using the two point correlation function $\xi(r)=\langle|\mathbf{B}(\mathbf{x}+\mathbf{r}) \mathbf{B}(\mathbf{x})|\rangle$ as

$$
L_{\mathrm{int}}=\frac{1}{\xi(0)} \int_{0}^{\infty} d r \xi(r) .
$$

We refer to this scale as the integral scale and define the comoving wave number of the integral scale as $k_{\text {int }} \equiv$ $a / L_{\text {int }}$.

The integral scale grows in time. In the turbulent regime a direct cascade due to nonlinear interactions transfers energy from the integral scale to the much smaller viscous scale, where it is lost to heat. In the free-streaming regime the integral scale may also grow due to the dissipation of flows on the integral scale itself. In both regimes the damping time scale is given by

$$
t_{\mathrm{eddy}} \approx \frac{L}{v} \approx \frac{a}{k v} .
$$

By comparing this eddy time scale on the integral scale with the cosmic time $H^{-1}$, we can judge whether nonlinear cascade processes are important or not. If once $t_{\text {eddy }}^{\text {int }} \equiv$ $L_{\text {int }} / v<H^{-1}$ is satisfied, magnetic energy dissipation becomes effective and magnetic energy on scales $L<L_{\text {int }}$ is lost.

Now let us classify the evolution of the MHD nonlinear Alfvén modes into three regimes, i.e., turbulent, viscous, and free-streaming regimes, following the results of the numerical simulation. In the following argument, we mostly consider the evolution of Alfvén modes at the integral scale.

\section{A. Turbulent regime}

In the early universe, since the photon mean free path $L_{\mathrm{mfp}}$, which is proportional to $1 / n_{e}$, is very short, the effect of viscosity on Alfvén modes at the integral scale is negligible until the mean free path becomes large enough to have Reynolds number $R \sim 1$ on $L_{\text {int }}$. For $R>1$ we can ignore the dissipation term $\mathbf{f}$ in Eq. (5). The advective term $\left(\mathbf{v}_{\mathrm{A}} \cdot \nabla\right) \mathbf{v}_{\mathrm{A}} / a$ drives the fluid velocity in this regime, which we call the turbulent regime.

Ignoring the cosmological expansion term, we find that the fluid velocity eventually approaches an equipartition state:

$$
\mathbf{v}=\mathbf{v}_{\mathrm{A}} .
$$

This behavior is consistent with the MHD numerical simulation $[10,11]$. Note that it is not clear whether the fluid velocity approaches an equipartition state on the scales larger than the integral scale or not since it takes longer on larger scales. We will discuss this point in Sec. III A.

If magnetic fields do not decay, the Alfvén velocity stays constant in time since $B \propto a^{-2}$ for the adiabatic expansion and $\rho_{\mathrm{t}} \propto a^{-4}$ during the radiation dominated epoch. Accordingly the eddy time scale at the integral scale evolves as $t_{\text {eddy }}^{\text {int }}=L_{\text {int }} / v_{\mathrm{A}} \propto L_{\text {int }} /\left(B / \sqrt{\rho_{\mathrm{t}}}\right) \propto a$. On the other hand, the cosmic time $1 / H$ is proportional to $a^{2}$ in the radiation dominated epoch. Therefore soon or later the cosmic time exceeds the eddy time, which allows for turbulent decay of the magnetic fields.

Let us go into details about the evolution of the integral scale. By definition the integral scale corresponds to the peak location of the energy power spectrum of the magnetic field. Correspondingly, the Alfvén velocity, which is proportional to the amplitude of the magnetic field, peaks at the integral scale. Since we assume a blue magnetic power spectrum, i.e. $n>0$, the eddy turnover time on the scales larger than the integral scale is longer than $t_{\text {eddy }}^{\text {int }}$. 
Therefore the condition $t_{\text {eddy }}=1 / H$ is first satisfied on the integral scale and then gradually moves towards larger scales. Meantime, the cascading decay of the short wavelength modes $\left(k>k_{\text {int }}\right)$ shifts the integral scale towards larger scales.

The underlying physical process of the cascade decay is following. When nonlinear effects become prominent, the magnetic field energy and the fluid kinetic energy achieve equipartition. The flow eddies break into the smaller eddies. Hence the kinetic energy is transported from large scales to small scales by a nonlinear cascade (Kolmogorov process). The transported energy is changed into heat at the scale where the dissipation process is effective. Consequently, the magnetic field energy at the integral scale is converted into heat which is called direct cascade.

\section{B. Viscous regime}

As the universe evolves, the photon mean free path, $L_{\mathrm{mfp}} \propto a^{3}$, becomes larger. Dissipation due to photon interaction becomes efficient and velocity fluctuations are damped by the photon drag. Eventually the dissipation term dominates the advective term at the integral scale in Eq. (5). This is the second regime, which is refereed as the viscous regime. During the viscous regime, the eddy time at the integral scale is always larger than the cosmic time due to the decay of the fluid velocity $v$, which makes the eddy time longer.

The transition epoch from turbulent to viscous regimes on the integral scale can be determined by comparing the advective term $(\mathbf{v} \cdot \nabla) \mathbf{v} / a$ and the dissipation term $\mathbf{f}=$ $\left(\nu /\left(\rho_{\mathrm{t}}+p_{\mathrm{t}}\right)\right) \nabla^{2} \mathbf{v} / a^{2}$. During the turbulent regime, the amplitude of the fluid velocity in the advective term is equal to the Alfvén velocity $v_{\mathrm{A}}$ as shown in the previous subsection. Therefore the advective term can be written by using the integral scale as $(\mathbf{v} \cdot \nabla) \mathbf{v} / a \simeq v_{\mathrm{A}}^{2} / L_{\text {int }}$. The dissipation term can be also rewritten as $\left(\nu /\left(\rho_{\mathrm{t}}+\right.\right.$ $\left.\left.p_{\mathrm{t}}\right)\right) v_{\mathrm{A}} / L_{\text {int }}^{2}$. At the transition epoch, these two terms become equal, which yields

$$
v_{\mathrm{A}}=\frac{\nu}{\left(\rho_{\mathrm{t}}+p_{\mathrm{t}}\right) L_{\mathrm{int}}}=\frac{L_{\mathrm{mfp}}}{5 L_{\mathrm{int}}} .
$$

Here we assume the radiation domination, i.e., $\rho_{\mathrm{t}}+p_{\mathrm{t}}=$ $4 \rho_{\gamma} / 3$. Until the beginning of the viscous regime, the eddy time $t_{\text {eddy }}=L_{\text {int }} / v_{\mathrm{A}}$ is equal to the cosmic time $1 / H$, which leads to $v_{\mathrm{A}} \simeq \sqrt{H /\left(n_{e} \sigma_{\mathrm{T}}\right)}$. Inserting the definition of $v_{\mathrm{A}}$ into this equation, the transition redshift $z_{\mathrm{t}-\mathrm{v}}$ can be obtained as

$$
z_{\mathrm{t}-\mathrm{v}} \simeq 6 \times 10^{6} B_{-9}^{-2} \simeq 6 \times 10^{6}\left(\frac{k_{\mathrm{int}}}{3.4 \times 10^{4} \mathrm{Mpc}^{-1}}\right)^{2 / 3},
$$

where $B_{-9}$ is the comoving magnetic field normalized by $10^{-9}$ Gauss, i.e., $B_{-9}=\left(B / 10^{-9}\right) a^{2}$ and

$$
k_{\mathrm{int}} \simeq 3.4 \times 10^{4} B_{-9}^{-3} \mathrm{Mpc}^{-1},
$$

is the comoving wave number of the integral scale at the transition epoch. Note that Eqs. (12) and (13) are only valid after $e^{ \pm}$annihilation where $L_{\mathrm{mfp}}=1 / \sigma_{\mathrm{T}} n_{e}$, i.e., $z<10^{8}$. For earlier epochs Eq. (11) must be evaluated numerically to find the redshift-B-field relations.

In the viscous regime, we can ignore the advective term in Eq. (5) at the integral scale since the fluid velocity $v$ decays due to the dissipation. We can also omit the expansion term since the evolution of the fluid velocity is controlled by the dissipation whose time scale is much faster than the cosmic expansion. Employing the terminalvelocity approximation, we obtain

$$
\frac{v_{\mathrm{A}}^{2}}{L_{\mathrm{int}}}=\frac{\nu}{\left(\rho_{\mathrm{t}}+p_{\mathrm{t}}\right)} \frac{v}{L_{\mathrm{int}}^{2}} .
$$

Using the comoving wave number at the integral scale $k_{\text {int }}$, the fluid velocity can be described as

$$
v=\left(\rho_{\mathrm{t}}+p_{\mathrm{t}}\right) \frac{v_{\mathrm{A}}^{2}}{\nu} \frac{a}{k_{\mathrm{int}}} .
$$

Hence it is found that the evolution of the fluid velocity becomes $v \propto a^{-2}$.

According to this solution, the eddy time at the integral scale is proportional to $a^{3}$ in both radiation and matter dominated epochs. Therefore the eddy time remains longer than the cosmic expansion time, $1 / H$ which is proportional to $a^{2}$, and $a^{3 / 2}$ in the radiation dominated epoch and the matter dominated epoch, respectively. As a result, no direct cascade occurs during the viscous regime and the integral scale does not grow.

\section{Free-streaming regime}

The third regime is the free-streaming regime, in which photon mean free path is larger than the integral scale. In this regime, photons and baryon fluids are decoupled and magnetic fields can amplify the fluid velocity. This amplification of the velocity makes the eddy time shorter until the eddy time becomes equal to the cosmic time $1 / H$. At this point the kinetic energy on $L_{\text {int }}$ is directly dissipated into heat and the integral scale shifts to larger scales.

Let us first estimate the transition epoch from the viscous regime to the free-streaming regime. The transition happens when the mean free path $L_{\mathrm{mfp}}$ becomes equal to the integral scale $L_{\text {int }}$. Employing the comoving wave number of the integral scale in the viscous regime Eq. (13), we obtain the transition redshift as

$$
\begin{aligned}
z_{\mathrm{v}-\mathrm{f}} & \simeq 1.7 \times 10^{5} B_{-9}^{-3 / 2} \\
& \simeq 1.7 \times 10^{5}\left(\frac{k}{3.4 \times 10^{4} \mathrm{Mpc}^{-1}}\right)^{1 / 2},
\end{aligned}
$$

Using Eq. (15), and noting that $a / k_{\text {int }} \approx L_{\mathrm{mfp}}$ during this epoch, we find that $v$ has decayed to $v \approx v_{A}^{2}$ at the tran- 
sition. In the free-streaming regime, we need to solve Eq. (7) instead of Eq. (5) for the evolution of the fluid velocity. Ignoring the cosmological expansion term, we obtain the solution with the terminal-velocity approximation as

$$
v=\frac{\left(\rho_{\mathrm{t}}+p_{\mathrm{t}}\right) k_{\mathrm{int}} v_{\mathrm{A}}^{2}}{\rho_{\mathrm{b}} \alpha a} .
$$

The eddy time is longer than the cosmic time when the free-streaming epoch begins since $v$ is much smaller than $v_{\mathrm{A}}$ during the viscous regime due to dissipation. From the above solution, however, it is found that $v \propto a^{2}$ in the freestreaming epoch. Accordingly the eddy time evolves as $a^{-1}$. Therefore the eddy time soon becomes shorter than the cosmic time which is proportional to $a^{2 / 3}$ in the matter dominated universe.

As is shown in the previous subsection, the integral scale does not evolve in the viscous regime. In the free-streaming regime, the integral scale does also not change until the eddy time becomes equal to the cosmic time.

When the eddy time is equal to the cosmic time on the integral scale, i.e. $L_{\text {int }} / v=1 / H$, the comoving wave number must satisfy the relation (using Eqs. (17) and (6)):

$$
k_{\mathrm{int}}^{-1} \simeq \frac{v_{\mathrm{A}}}{k_{\mathrm{S}}},
$$

where $k_{\mathrm{S}}^{-1}$ is the comoving Silk scale defined as $k_{\mathrm{S}}^{-1} \simeq$ $\sqrt{L_{\mathrm{mfp}} / H} / a$. Note that in this regime, $v_{\mathrm{A}} / k_{\mathrm{S}}$ is the comoving damping scale for Alfvén modes. Unlike in the nonmagnetized photon-baryon fluid overdamped Alfvén modes survive below the Silk damping scale for weak magnetic fields as was first pointed out by $[7,8]$. While it is not clear either the direct cascade or the diffusion process takes place first, the baryon velocity exponentially damps away bellow this scale.

Substituting Eq. (13), which is the comoving integral wave number in the viscous regime and in the freestreaming regime until the damping scale for Alfvén modes, $v_{\mathrm{A}} / k_{\mathrm{S}}$, becomes equal to the integral scale, into Eq. (18), we obtain the redshift below which further growth of the integral scale in the (viscous) free-streaming regime occurs

$$
z=1100\left(\frac{B_{-9}}{16 \text { nGauss }}\right)^{-8 / 5}=1100\left(\frac{k_{\text {int }}}{8 \mathrm{Mpc}^{-1}}\right)^{8 / 15} .
$$

Therefore if the integral scale once became larger than $100 \mathrm{kpc}$ by the end of the turbulent regime, the integral scale did not change through viscous and free-streaming regimes all the way until the recombination epoch.

On the contrary, if the integral scale was smaller than $100 \mathrm{kpc}$, there was further growth of $L_{\text {int }}$ during the freestreaming regime before recombination. The integral scale shifts to a larger scale in this case. The resultant integral

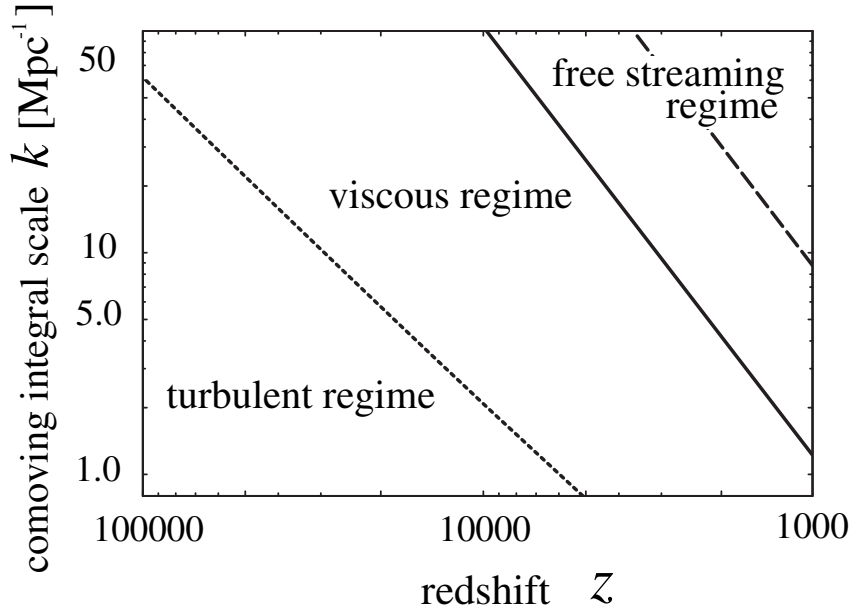

FIG. 1. The transition comoving scales as the function of the redshift. The dotted line is the transition line from the turbulent to the viscous regime, Eq. (12). The solid line is that from the viscous to the free-streaming regime, Eq. (16). The dashed line represents the start of the direct cascade in the free-streaming regime, Eq. (19), which is more or less when the diffusion process takes place. In the left side of this line there is no direct cascade or diffusion damping. In the right side the direct cascade or diffusion damping is occurring.

scale at the recombination epoch depends on the slope of the magnetic field power spectrum.

In Fig. 1 we show the areas of turbulent, viscous and free-streaming regimes in the $z-k_{\text {int }}$ plane. Here we employ Eqs. (12), (16), and (19). Knowing the time evolution of the integral scale $k_{\text {int }}$, which depends on the magnetic field spectrum, we can draw an evolutionary track in this plane and easily understand when the transition between different regimes occurs. We will discuss this evolution in the next section.

\section{ALFVÉN MODE SPECTRUM}

In the previous section, we discussed the evolution history of magnetic fields and fluid velocity or Alfvén modes. We separated the evolution into three regimes, i.e., turbulent, viscous, and free-streaming. We analytically solved the MHD-Euler equation and acquired the relation between the fluid velocity $v$ and the Alfvén velocity $v_{\mathrm{A}}$ in each regime. Once $v$ was obtained, we could estimate the eddy time $t_{\text {eddy }}$ and compare it with the cosmic time in order to know whether field evolution occurred.

However we can not describe the time evolution of the integral scale of the magnetic fields without knowing the magnetic field power spectrum.

In this section, we find the power spectra of magnetic fields and the fluid velocities for a given initial shape of the magnetic field power spectrum. Being motivated by a causal mechanism of primordial magnetic field generation such as QCD phase transition, we employ the power law 
spectrum with exponential cutoff for initial comoving magnetic fields as

$$
\begin{aligned}
\mathcal{P}_{B}^{\text {comov }}\left(t_{\mathrm{i}}, k\right) & =\frac{k^{3}}{2 \pi^{2}}\left\langle\left|B_{\text {comov }}\left(t_{\mathrm{i}}, k\right)\right|^{2}\right\rangle \\
& =B_{\text {comov }}^{2}\left(\frac{k}{k_{\mathrm{c}}}\right)^{n} \exp \left(-\left(\frac{k}{k_{\mathrm{c}}}\right)^{2}\right),
\end{aligned}
$$

where $n$ is the initial power law index and $k_{\mathrm{c}}$ is the cutoff scale, and $B_{\text {comov }}$ is the amplitude of the initial comoving magnetic fields at the cutoff scale. Obviously, the cutoff scale corresponds to the integral scale at the initial epoch for a blue, $n>0$ spectra.

To see the evolution of the fluid velocity induced by the magnetic field, it is convenient to define the power spectrum of the Alfvén velocity $\mathcal{P}_{\mathrm{A}}(k)$ as

$$
\begin{aligned}
\mathcal{P}_{\mathrm{A}}(t, k) & \equiv \frac{k^{3}}{2 \pi^{2}}\left\langle\left|v_{\mathrm{A}}(t, k)\right|^{2}\right\rangle \\
& =\frac{1}{4 \pi} \frac{1}{\left(\rho_{\mathrm{t}}+p_{\mathrm{t}}\right) a^{4}} \mathcal{P}_{B}^{\text {comov }}(t, k) .
\end{aligned}
$$

\section{A. Turbulent regime}

In the turbulent regime, we have shown that the magnetic field energy reaches an equipartition state with the fluid kinetic energy, i.e., $v=v_{\mathrm{A}}$ (Eq. (10)).

Therefore we expect to have the following relation between the power spectrum of the fluid velocity $\mathcal{P}_{v}(t, k)$ and the energy density of the magnetic field, i.e. the power spectrum of the Alfvén velocity, $\mathcal{P}_{\mathrm{A}}(t, k)$, as

$$
\mathcal{P}_{\mathrm{A}}(t, k)=\mathcal{P}_{v}(t, k) .
$$

Here the power spectrum of the fluid velocity is defined as

$$
\mathcal{P}_{v}(k)=\frac{k^{3}}{2 \pi^{2}}\left\langle|v|^{2}\right\rangle
$$

Equation (22) is consistent with the results of the MHD simulation as is shown in Fig. 2. In this figure $s$ is the time stamp and has the following relation with the redshift $z$,

$$
\frac{z+1}{z_{\mathrm{i}}+1}=\exp \left(-s \frac{t_{\text {ieddy }}}{H_{0}^{-1}}\right)
$$

where the subscript $i$ denotes the initial value. Initially, the power spectrum of the fluid velocity is very different from the one of the magnetic field. However, the fluid velocity power spectrum soon catches up with the magnetic field one. Subsequently, they evolve in a very similar manner. In this figure, it is shown fluid velocity on large scales also immediately reaches the equipartition as well as the velocity on scales smaller than the integral scale. Therefore hereafter we assume the equipartition on all scales, i.e., Eq. (22). Although it is immediate in terms of $s$, however, it may take longer time in physical time. If this is the case and the fluid velocity on larger scales has not caught up with the Alfvén velocity by the end of the turbulent regime, we expect that the power spectrum of the fluid velocity $\mathcal{P}_{v}(k)$ has less power on large scales than the one of the magnetic fields $\mathcal{P}_{\mathrm{A}}(k)$ which is proportional to $k^{n}$ on large scales. The most extreme case is that turbulence does not work at all on large scales, in which we need to employ linear perturbation. Subramanian and Barrow [16] obtain $\mathcal{P}_{v}(k) \propto k^{5}$ for the linear calculation. Therefore the power law slope on large scales can be between $n$ and 5 .

On scales larger than the integral scale, the power spectrum of the Alfvén velocity keeps its initial amplitude. The power spectrum of the fluid velocity also soon reaches to this amplitude.

Let us now estimate the evolution of the integral scale. In Sec. II A, we explained the reason why this scale shifts to larger scale with time during the turbulent regime. Now we would like to estimate the rate of this shift as a function of the power law index $n$.

As we have shown in II A, the integral scale is the scale where $t_{\text {eddy }}=1 / H$ is satisfied. In the turbulent regime $t_{\text {eddy }}=a /\left(k_{\text {int }} v\right)=a /\left(k_{\text {int }} v_{\mathrm{A}}\right)$. Therefore $k_{\text {int }}=a H / v_{\mathrm{A}}$. The time evolution of the Alfvén velocity at the integral scale, in the absence of dissipation, is estimated as $v_{\mathrm{A}}=$ $B / \sqrt{\rho_{\mathrm{t}}+p_{\mathrm{t}}} \propto \sqrt{\mathcal{P}_{\mathrm{A}}\left(k_{\text {int }}\right)} \times a^{0}$. On the scale larger than the previous integral scale, $\mathcal{P}_{B}(k) \propto k^{n}$. Therefore the growth of the integral scale, when dissipation is also considered, is obtained as

$$
k_{\text {int }} \propto a^{-2 /(n+2)} .
$$

This analytic estimate is supported by the numerical simulation as is seen in Fig. 2. This figure also shows the direct

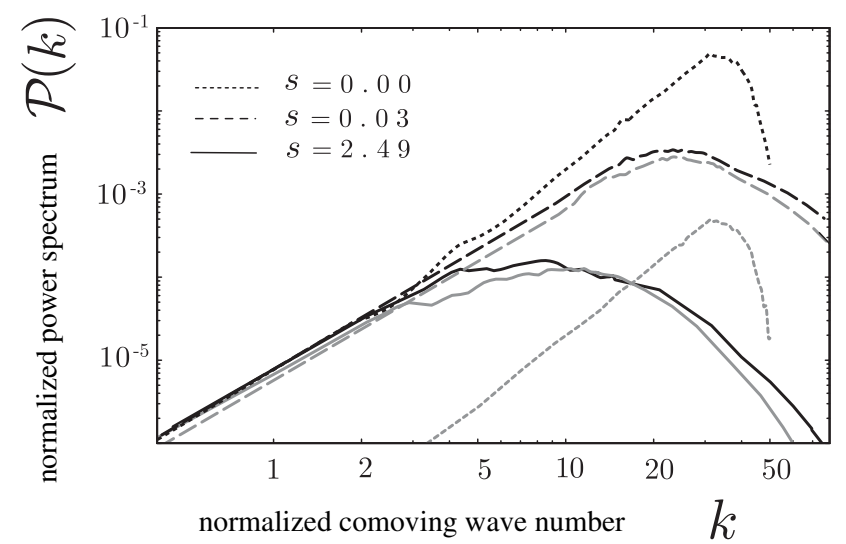

FIG. 2. The evolution of the comoving energy power spectrum of the magnetic fields and the kinetic energy power spectrum of the fluids in the turbulent regime from the numerical simulation by $[10,11]$. The $\mathrm{x}$-axis is a comoving wave number. The normalizations of $\mathrm{x}$ and $\mathrm{y}$-axes are arbitrary. The thin and thick lines are the power spectra of magnetic fields and fluids, respectively. The dotted lines are the initial power spectra $(\tau=0.00)$ with the spectrum index $n=4$. This figure shows that the energy densities become the equipartition, $v=v_{\mathrm{A}}$ after a few eddy time scale even on large scales. 
cascade of the magnetic fields soon smears out the initial sharp damping in the power spectrum.

The resultant power spectrum of the magnetic fields during the turbulent regime has power law shape on both large and small scales but with different indices. Matching the amplitude with the initial value on large scales, we obtain

$$
\begin{gathered}
\mathcal{P}_{\mathrm{A}}(t, k)=\mathcal{P}_{\mathrm{A}}\left(t_{\mathrm{i}}, k\right) \propto k^{n}, \quad k<k_{\text {int }}, \\
\mathcal{P}_{\mathrm{A}}(t, k)=\mathcal{P}_{\mathrm{A}}\left(t_{\mathrm{i}}, k_{\text {int }}\right)\left(\frac{k}{k_{\text {int }}}\right)^{m} \propto k^{m}, \quad k>k_{\text {int }},
\end{gathered}
$$

where $m$ as observed in the numerical simulation is approximately $m \sim-2 / 3$ (for details see [10]).

Because of the equipartition, the evolution of the power spectrum of the fluid velocity is identical to the Alfvén velocity as

$$
\begin{gathered}
\mathcal{P}_{v}(t, k)=\mathcal{P}_{\mathrm{A}}\left(t_{\mathrm{i}}, k\right) \propto k^{n}, \quad k<k_{\text {int }}, \\
\mathcal{P}_{v}(t, k)=\mathcal{P}_{\mathrm{A}}\left(t_{\mathrm{i}}, k_{\text {int }}\right)\left(\frac{k}{k_{\text {int }}}\right)^{m} \propto k^{m}, \quad k>k_{\text {int }} .
\end{gathered}
$$

\section{B. Viscous regime}

When the relation $v_{\mathrm{A}}=\left(\rho_{\mathrm{t}}+p_{\mathrm{t}}\right) \nu k / a$ is satisfied at the integral scale, the magnetic fields enter into the viscous regime. In the viscous regime the magnetic fields do not decay. Therefore the spectrum of the magnetic fields is frozen over the viscous regime and keeps its feature at the transition epoch from the turbulent to the viscous regimes $t_{\mathrm{t}-\mathrm{v}}$ as

$$
\mathcal{P}_{\mathrm{A}}(t, k)=\mathcal{P}_{\mathrm{A}}\left(t_{\mathrm{t}-\mathrm{v}}, k\right) .
$$

In the viscous regime no further growth of $L_{\text {int }}$ happens and the amplitude of the fluid velocity is proportional to square of the Alfvén velocity amplitude as is shown in Eq. (15). Note that Eq. (15) can be applied not only to the integral scale but also to all scales during the viscous regime. Therefore the power spectrum of the fluid velocity can be described using the power spectrum of the Alfvén velocity.

If the power law index $n$ is smaller than $3 / 2$, the contribution from larger $k$ (smaller scales) is negligible and we obtain

$$
\begin{aligned}
\mathcal{P}_{v}(t, k) & \approx\left(\frac{\left(\rho_{\mathrm{t}}+p_{\mathrm{t}}\right) a}{\nu k} \mathcal{P}_{\mathrm{A}}\left(t_{\mathrm{t}-\mathrm{v}}, k\right)\right)^{2} \\
& \propto \begin{cases}k^{2(n-1)}, & k<k_{\mathrm{int}}, \\
k^{2(m-1)}, & k>k_{\text {int }} .\end{cases}
\end{aligned}
$$

If $n$ is larger than $3 / 2$, on the other hand, the contribution from the integral scale dominates in the convolution on scales larger than the integral scale. Then we can simply describe the power spectrum of the fluid velocity as (cf.
Appendix A, Eq. (A23) and text below)

$$
\begin{aligned}
& \mathcal{P}_{v}(t, k) \approx\left(\frac{\left(\rho_{\mathrm{t}}+p_{\mathrm{t}}\right) a}{\nu k_{\mathrm{int}}} \mathcal{P}_{\mathrm{A}}\left(t_{\mathrm{t}-\mathrm{v}}, k_{\mathrm{int}}\right)\right)^{2}\left(\frac{k}{k_{\mathrm{int}}}\right) \propto k, \quad k<k_{\mathrm{int}}, \\
& \mathcal{P}_{v}(t, k) \approx\left(\frac{\left(\rho_{\mathrm{t}}+p_{\mathrm{t}}\right) a}{\nu k} \mathcal{P}_{\mathrm{A}}\left(t_{\mathrm{t}-\mathrm{v}}, k\right)\right)^{2} \propto k^{2(m-1)}, \quad k>k_{\mathrm{int}} .
\end{aligned}
$$

Note that in Eq. (32), the slope may be steeper on large scales if the equipartition was not achieved during the turbulent regime.

On scales smaller than the photon mean free path $L_{\mathrm{mfp}}$, the diffusion approximation is no longer valid as is the case in the free-streaming regime regardless of the value of the initial power law index $n$. Therefore we shall use the argument of Sec. II C for the evolution of the fluid velocity. Employing Eq. (17), we obtain

$$
\mathcal{P}_{v}(t, k) \approx\left(\frac{\left(\rho_{\mathrm{t}}+p_{\mathrm{t}}\right) k}{\rho_{\mathrm{b}} \alpha a} \mathcal{P}_{\mathrm{A}}\left(t_{\mathrm{t}-\mathrm{v}}, k\right)\right)^{2} \propto k^{2(m+1)}, \quad k>k_{\mathrm{f}},
$$

where $k_{\mathrm{f}} \equiv \sqrt{5} a / L_{\mathrm{mfp}}$. For the definition of $k_{\mathrm{f}}$, we put the factor $\sqrt{5}$ in order to match the power spectrum at $k_{\mathrm{f}}$. The estimated value of $k_{\mathrm{f}}$ at the recombination epoch is $k_{\mathrm{f}}=$ $1.2 \mathrm{Mpc}^{-1}(\mathrm{z} / 1100)^{2}$.

\section{Free-streaming regime}

When the integral scale becomes smaller than the photon mean free path, i.e., $k_{\mathrm{f}}=k_{\text {int }}$, the free-streaming regime begins. At the beginning of the free-streaming regime, the eddy time at the integral scale is longer than the cosmic time. Therefore the integral scale does not change. If the integral scale is larger than $100 \mathrm{kpc}$, the eddy time is always longer than the cosmic time until recombination as is described in Eq. (19), and further dissipation never occurs. In this case, the Alfvén velocity $v_{\mathrm{A}}$ does not evolve and the fluid velocity is expressed in terms of $v_{\mathrm{A}}$ as $v=\left(\rho_{\mathrm{t}}+p_{\mathrm{t}}\right) k v_{\mathrm{A}}^{2} /\left(\rho_{\mathrm{b}} \alpha a\right)$ (Eq. (17)). Accordingly the power spectrum of the fluid velocity can be described by the convolution of the power spectrum of the Alfvén velocity.

Following the argument we made in the previous subsection, we obtain the power spectrum of the fluid velocity as

$$
\begin{aligned}
\mathcal{P}_{v}(t, k) & \approx\left(\frac{\left(\rho_{\mathrm{t}}+p_{\mathrm{t}}\right) k}{\rho_{\mathrm{b}} \alpha a} \mathcal{P}_{\mathrm{A}}\left(t_{\mathrm{v}-\mathrm{f}}, k\right)\right)^{2} \\
& \propto \begin{cases}k^{2(n+1)}, & k_{\mathrm{f}}<k<k_{\mathrm{int}}, \\
k^{2(m+1)}, & k>k_{\mathrm{int}},\end{cases}
\end{aligned}
$$

for the power law index $n$ being smaller than $3 / 2$. 
If $n>3 / 2$, on the contrary, we obtain

$$
\begin{aligned}
\mathcal{P}_{v}(t, k) & \approx\left(\frac{\left(\rho_{\mathrm{t}}+p_{\mathrm{t}}\right) k_{\mathrm{int}}}{\rho_{\mathrm{b}} \alpha a} \mathcal{P}_{\mathrm{A}}\left(t_{\mathrm{t}-\mathrm{v}}, k_{\mathrm{int}}\right)\right)^{2}\left(\frac{k}{k_{\mathrm{int}}}\right)^{5} \propto k^{5} \\
k_{\mathrm{f}} & <k<k_{\mathrm{int}} \\
\mathcal{P}_{v}(t, k) & \approx\left(\frac{\left(\rho_{\mathrm{t}}+p_{\mathrm{t}}\right) k}{\rho_{\mathrm{b}} \alpha a} \mathcal{P}_{\mathrm{A}}\left(t_{\mathrm{v}-\mathrm{f}}, k\right)\right)^{2} \propto k^{2(m+1)}, \quad k>k_{\mathrm{int}} .
\end{aligned}
$$

On the scales larger than the free-streaming scale, i.e., $k<k_{\mathrm{f}}$, the evolution of the fluid velocity still follows the solution of the viscous regime. Therefore

$$
\begin{aligned}
\mathcal{P}_{v}(t, k) & \approx\left(\frac{\left(\rho_{\mathrm{t}}+p_{\mathrm{t}}\right) a}{\nu k_{\mathrm{int}}} \mathcal{P}_{\mathrm{A}}\left(t_{\mathrm{t}-\mathrm{v}}, k_{\mathrm{int}}\right)\right)^{2}\left(\frac{k}{k_{\mathrm{int}}}\right) \propto k, \\
k & <k_{\mathrm{f}}, \quad n>3 / 2, \\
\mathcal{P}_{v}(t, k) & \approx\left(\frac{\left(\rho_{\mathrm{t}}+p_{\mathrm{t}}\right) a}{\nu k} \mathcal{P}_{\mathrm{A}}\left(t_{\mathrm{t}-\mathrm{v}}, k\right)\right)^{2} \propto k^{2(n-1)}, \\
k & <k_{\mathrm{f}}, \quad n<3 / 2 .
\end{aligned}
$$

Note that in Eqs. (38) and (39), the slopes may be steeper on large scales as is pointed out in the previous subsection.

Finally, in the case of the integral scale at the transition epoch from the viscous to free-streaming regimes being smaller than $100 \mathrm{kpc}$, the further processing of $L_{\text {int }}$ begins at the redshift of Eq. (19). Once further decay takes place, the integral scale shifts to larger scales. Let us now estimate the evolution of the integral scale as a function of the initial power law index $n$. The integral scale can be written as $k_{\text {int }}=a H / v$. The time evolution of the fluid velocity at the integral scale is estimated as $v=\left(\rho_{\mathrm{t}}+\right.$ $\left.p_{\mathrm{t}}\right) k v_{\mathrm{A}}^{2} /\left(\rho_{\mathrm{b}} \alpha a\right) \propto k_{\text {int }} \mathcal{P}_{\mathrm{A}}\left(k_{\text {int }}\right) a^{2}$. On the scale larger than the previous integral scale, $\mathcal{P}_{\mathrm{A}}(k) \propto k^{n}$. Therefore the time evolution of the integral scale is given by

$$
\begin{aligned}
& k_{\text {int }} \propto a^{-3 /(n+2)} \quad \text { in the radiation dominant, } \\
& k_{\text {int }} \propto a^{-5 /(2(n+2))} \quad \text { in the matter dominant. }
\end{aligned}
$$

We should note that $H \propto a^{-2}$ in the radiation dominated universe and $H \propto a^{-3 / 2}$ in the matter dominated universe while $\rho_{\mathrm{t}} \propto a^{-4}$ until the energy density of baryons dominate the one of photons, which happens much later than recombination. Therefore $v_{\mathrm{A}}=B / \sqrt{\rho_{\mathrm{t}}+p_{\mathrm{t}}}$ is still constant through this regime. This time evolution is consistent with the numerical result.

Now we can estimate the power spectrum on scales where the direct cascade or diffusion damping during the free-streaming regime occurs. Even if the cascade process occurs first, the power law slope of the decay immediately washes away by diffusion since the scales of the direct cascade and the diffusion are very close. Therefore we only consider the diffusion process here. The resultant power spectrum leads to

$$
\mathcal{P}_{v}(t, k) \approx\left(\frac{\left(\rho_{\mathrm{t}}+p_{\mathrm{t}}\right) k_{\text {int }}}{a \alpha \rho_{\mathrm{b}}} \mathcal{P}_{\mathrm{A}}\left(k_{\mathrm{int}}\right)\right)^{2} \mathrm{e}^{-2\left(k / k_{\text {int }}\right)^{2}}, \quad k>k_{\text {int }} .
$$

In the above power spectrum, the shape on the scales larger than the integral scale is identical to the one without further growth of the integral scale during free-streaming (cf. Eqs. (35)-(39)).

\section{Summary of velocity power spectra}

Let us summarize the fluid velocity power spectrum of Alfvén modes at LSS for estimating power spectra of CMB anisotropies and polarization. There are three different cases corresponding to the integral scale on LSS. We refer them as "Case A", "Case B" and "Case C" for $k_{\text {int }}<$ $k_{\mathrm{f}}=1.2 \mathrm{Mpc}^{-1}, k_{\mathrm{f}}=1.2 \mathrm{Mpc}^{-1}<k_{\text {int }}<8 \mathrm{Mpc}^{-1}$, and $8 \mathrm{Mpc}^{-1}<k_{\text {int }}$, respectively, (cf. to Fig. 1 at $z \approx 1000$ ). Here $1.2 \mathrm{Mpc}^{-1}$ and $8 \mathrm{Mpc}^{-1}$ correspond to amplitudes of comoving magnetic field strength as 30 nGauss and 16 nGauss. Case A is the case in which the viscous regime continues until recombination. For Case B, while transition from the viscous to the free-streaming regimes occurs before recombination, the direct cascade process never happens on the integral scale. The diffusion process on the integral scale only takes place for Case C.

Let us first list the power spectra with $n>3 / 2$.

Case A-(Eqs. (32)-(34))

$\mathcal{P}_{v}(t, k) \approx\left(\frac{\left(\rho_{\mathrm{t}}+p_{\mathrm{t}}\right) a}{\nu k_{\mathrm{int}}} \mathcal{P}_{\mathrm{A}}\left(t_{\mathrm{t}-\mathrm{v}}, k_{\mathrm{int}}\right)\right)^{2}\left(\frac{k}{k_{\mathrm{int}}}\right) \propto k, \quad k<k_{\mathrm{int}}$,

$\begin{aligned} \mathcal{P}_{v}(t, k) & \approx\left(\frac{\left(\rho_{\mathrm{t}}+p_{\mathrm{t}}\right) a}{\nu k} \mathcal{P}_{\mathrm{A}}\left(t_{\mathrm{t}-\mathrm{v}}, k\right)\right)^{2} \propto k^{2(m-1)}, \\ k_{\text {int }} & <k<k_{\mathrm{f}},\end{aligned}$

$$
\mathcal{P}_{v}(t, k) \approx\left(\frac{\left(\rho_{\mathrm{t}}+p_{\mathrm{t}}\right) k}{\rho_{\mathrm{b}} \alpha a} \mathcal{P}_{\mathrm{A}}\left(t_{\mathrm{t}-\mathrm{v}}, k\right)\right)^{2} \propto k^{2(m+1)}, \quad k_{\mathrm{f}}<k
$$

Case B-(Eqs. (38), (36), and (37))

$$
\mathcal{P}_{v}(t, k) \approx\left(\frac{\left(\rho_{\mathrm{t}}+p_{\mathrm{t}}\right) a}{\nu k_{\text {int }}} \mathcal{P}_{\mathrm{A}}\left(t_{\mathrm{t}-\mathrm{v}}, k_{\text {int }}\right)\right)^{2}\left(\frac{k}{k_{\text {int }}}\right) \propto k, \quad k<k_{\mathrm{f}},
$$

$$
\begin{aligned}
\mathcal{P}_{v}(t, k) & \approx\left(\frac{\left(\rho_{\mathrm{t}}+p_{\mathrm{t}}\right) k_{\text {int }}}{\rho_{\mathrm{b}} \alpha a} \mathcal{P}_{\mathrm{A}}\left(t_{\mathrm{t}-\mathrm{v}}, k_{\mathrm{int}}\right)\right)^{2}\left(\frac{k}{k_{\mathrm{int}}}\right)^{5} \propto k^{5}, \\
k_{\mathrm{f}} & <k<k_{\text {int }}, \\
\mathcal{P}_{v}(t, k) & \approx\left(\frac{\left(\rho_{\mathrm{t}}+p_{\mathrm{t}}\right) k}{\rho_{\mathrm{b}} \alpha a} \mathcal{P}_{\mathrm{A}}\left(t_{\mathrm{v}-\mathrm{f}}, k\right)\right)^{2} \propto k^{2(m+1)}, \quad k_{\mathrm{int}}<k .
\end{aligned}
$$


Case $C$-(Eqs. (38), (36), and (41))

$\mathcal{P}_{v}(t, k) \approx\left(\frac{\left(\rho_{\mathrm{t}}+p_{\mathrm{t}}\right) a}{\nu k_{\mathrm{int}}} \mathcal{P}_{\mathrm{A}}\left(t_{\mathrm{t}-\mathrm{v}}, k_{\mathrm{int}}\right)\right)^{2}\left(\frac{k}{k_{\mathrm{int}}}\right) \propto k, \quad k<k_{\mathrm{f}}$,

$\begin{aligned} \mathcal{P}_{v}(t, k) & \approx\left(\frac{\left(\rho_{\mathrm{t}}+p_{\mathrm{t}}\right) k_{\text {int }}}{\rho_{\mathrm{b}} \alpha a} \mathcal{P}_{\mathrm{A}}\left(t_{\mathrm{t}-\mathrm{v}}, k_{\mathrm{int}}\right)\right)^{2}\left(\frac{k}{k_{\text {int }}}\right)^{5} \propto k^{5}, \\ k_{\mathrm{f}} & <k<k_{\text {int }},\end{aligned}$

$\mathcal{P}_{v}(t, k) \approx\left(\frac{\left(\rho_{\mathrm{t}}+p_{\mathrm{t}}\right) k_{\text {int }}}{a \alpha \rho_{\mathrm{b}}} \mathcal{P}_{\mathrm{A}}\left(k_{\text {int }}\right)\right)^{2} \mathrm{e}^{-2\left(k / k_{\text {int }}\right)^{2}}, \quad k_{\text {int }}<k$

Fig. 3 shows the evolutionary track of each case. The Case A is represented as the dotted line. The Case B and Case $\mathrm{C}$ are the solid and the dashed lines. The slope of the tracks follow Eqs. (25) and (40). Here we employ the spectral index of the initial magnetic fields $n=4$.

Next we list the power spectra with $n<3 / 2$.

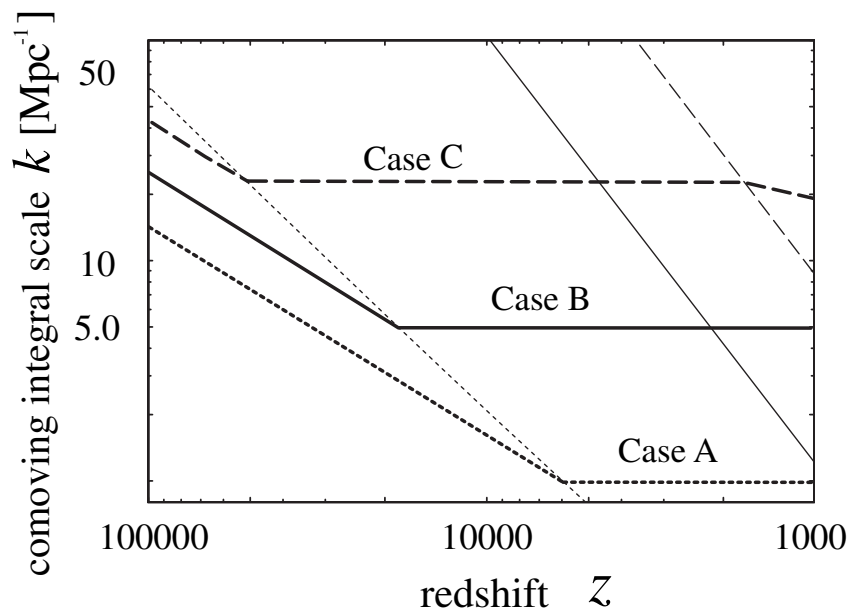

FIG. 3. Evolution of the comoving integral scale of the magnetic field configurations with $n=4$. The dotted line corresponds to Case A which undergoes the turbulent regime and the viscous regime before recombination. The solid line corresponds to Case B which passes through turbulent, viscous and free-streaming regimes, with nevertheless no growth of the integral scale immediately before recombination occurring. The dashed line corresponds to Case $\mathrm{C}$ in which there is growth of $L_{\text {int }}$ in the free-streaming regime shortly before recombination. The thin dotted line represents the transition line from the turbulent regime to the viscous regime. The thin solid line shows the evolution of $k_{\mathrm{f}}$, whereas the thin dashed line represents the start of growth of the integral scale in the free-streaming regime.
Case A'-(Eqs. (31) and (34))

$$
\begin{aligned}
& \mathcal{P}_{v}(t, k) \approx\left(\frac{\left(\rho_{\mathrm{t}}+p_{\mathrm{t}}\right) a}{\nu k} \mathcal{P}_{\mathrm{A}}\left(t_{\mathrm{t}-\mathrm{v}}, k\right)\right)^{2} \\
& \propto \begin{cases}k^{2(n-1)}, & k<k_{\mathrm{int}}, \\
k^{2(m-1)}, & k_{\mathrm{int}}<k<k_{\mathrm{f}},\end{cases} \\
& \mathcal{P}_{v}(t, k) \approx\left(\frac{\left(\rho_{\mathrm{t}}+p_{\mathrm{t}}\right) k}{\rho_{\mathrm{b}} \alpha a} \mathcal{P}_{\mathrm{A}}\left(t_{\mathrm{t}-\mathrm{v}}, k\right)\right)^{2} \propto k^{2(m+1)}, \quad k_{\mathrm{f}}<k .
\end{aligned}
$$

Case B'-(Eqs. (39) and (35))

$$
\mathcal{P}_{v}(t, k) \approx\left(\frac{\left(\rho_{\mathrm{t}}+p_{\mathrm{t}}\right) a}{\nu k} \mathcal{P}_{\mathrm{A}}\left(t_{\mathrm{t}-\mathrm{v}}, k\right)\right)^{2} \propto k^{2(n-1)}, \quad k<k_{\mathrm{f}},
$$

$$
\begin{aligned}
\mathcal{P}_{v}(t, k) & \approx\left(\frac{\left(\rho_{\mathrm{t}}+p_{\mathrm{t}}\right) k}{\rho_{\mathrm{b}} \alpha a} \mathcal{P}_{\mathrm{A}}\left(t_{\mathrm{v}-\mathrm{f}}, k\right)\right)^{2} \\
& \propto \begin{cases}k^{2(n+1)}, & k_{\mathrm{f}}<k<k_{\mathrm{int}}, \\
k^{2(m+1)}, & k_{\mathrm{int}}<k .\end{cases}
\end{aligned}
$$

Case C'-(Eqs. (39) and (35) $\left(k_{\mathrm{f}}<k<k_{\text {int }}\right)$ and (41))

$$
\mathcal{P}_{v}(t, k) \approx\left(\frac{\left(\rho_{\mathrm{t}}+p_{\mathrm{t}}\right) a}{\nu k} \mathcal{P}_{\mathrm{A}}\left(t_{\mathrm{t}-\mathrm{v}}, k\right)\right)^{2} \propto k^{2(n-1)}, \quad k<k_{\mathrm{f}},
$$

$$
\begin{aligned}
\mathcal{P}_{v}(t, k) & \approx\left(\frac{\left(\rho_{\mathrm{t}}+p_{\mathrm{t}}\right) k}{\rho_{\mathrm{b}} \alpha a} \mathcal{P}_{\mathrm{A}}\left(t_{\mathrm{v}-\mathrm{f}}, k\right)\right)^{2} \propto k^{2(n+1)}, \\
k_{\mathrm{f}} & <k<k_{\text {int }}, \\
\mathcal{P}_{v}(t, k) & \approx\left(\frac{\left(\rho_{\mathrm{t}}+p_{\mathrm{t}}\right) k_{\text {int }}}{a \alpha \rho_{\mathrm{b}}} \mathcal{P}_{\mathrm{A}}\left(k_{\text {int }}\right)\right)^{2} \mathrm{e}^{-2\left(k / k_{\text {int }}\right)^{2}}, \quad k_{\text {int }}<k
\end{aligned}
$$

Note that on large scales $\left(k<k_{\text {int }}\right)$, the power law slopes of above equations may be steeper as is pointed out before. In the most extreme case, $\mathcal{P}_{v}(t, k) \propto k^{5}$ instead of $k$ for $n>$ $3 / 2$, and $\mathcal{P}_{v}(t, k) \propto k^{2(n+1)}$ instead of $k^{2(n-1)}$ for $n<3 / 2$ as is expected from the linear analysis.

\section{CALCULATION OF CMB TEMPERATURE ANISOTROPY AND POLARIZATION SPECTRA}

Alfvén modes of baryon-electron fluid produced by the primordial magnetic fields generate $\mathrm{CMB}$ temperature fluctuations by a Doppler shift [16]. Moreover, the quadrupole component of the generated temperature anisotropies produces polarization due to Thomson scattering. It is known that $\mathrm{CMB}$ polarization can be decomposed into two parity independent modes, i.e., the E-mode (electric type) and the B-mode (magnetic type) [21]. Among them, the Bmode polarization is not primarily produced by the scalar type perturbations which eventually form the structure of 
the universe and provide dominant contribution on the temperature anisotropies. Therefore the B-mode polarization possibly can be a good probe of primordial magnetic fields.

First, we summarize the derivations of the temperature anisotropy and polarization spectra induced by Alfvén modes with use of the linear perturbation theory $[16,17,22]$. The Alfvén mode in the linear MHD theory is identical to the vector mode of the cosmological fluid velocity in the cosmological perturbations. The vector metric perturbations in the linear perturbation theory can be written as (cf. [26] also for notation)

$$
\begin{aligned}
& \delta g_{0 i}=-a^{2} V_{i}=-a^{2} V Q_{i}, \\
& \delta g_{i j}=2 a^{2} H_{T i j}=2 a^{2} H_{T} Q_{i j},
\end{aligned}
$$

where $V$ and $H_{T}$ are the amplitudes of the vector metric perturbations and $Q_{i}$ and $Q_{i j}$ are the vector type mode functions which are defined as

$$
\begin{aligned}
\nabla^{2} Q_{i} & =-k^{2} Q_{i}, \quad \nabla^{i} Q_{i}=0, \\
Q_{i j} & =-\frac{1}{2 k}\left(\nabla_{i} Q_{j}+\nabla_{j} Q_{i}\right) .
\end{aligned}
$$

The amplitude $v_{\mathrm{b}}$ of the vector components of the baryon fluid velocity $v_{\mathrm{b} i}$ can be represented with the mode functions as

$$
v_{\mathrm{b} i}=v_{\mathrm{b}} Q_{i} .
$$

Employing the total angular momentum method [27], we obtain the CMB temperature anisotropies $\Delta T^{T T}(l)$ induced by Alfvén modes as

$$
\begin{aligned}
& \Delta T^{T T}(l)=T_{0} \sqrt{\frac{l(l+1) C^{T T}(l)}{2 \pi}}, \\
& \Delta T^{B B}(l)=T_{0} \sqrt{\frac{l(l+1) C^{B B}(l)}{2 \pi}}, \\
& C^{T T}(l)=\frac{4}{\pi} \int_{0}^{\infty} k^{2} d k \quad\left\langle\left|\frac{\Theta_{l}^{(1)}\left(\eta_{0}, k\right)}{2 l+1}\right|^{2}\right\rangle, \\
& C^{B B}(l)=\frac{4}{\pi} \int_{0}^{\infty} k^{2} d k\left\langle\left|\frac{B_{l}^{(1)}\left(\eta_{0}, k\right)}{2 l+1}\right|^{2}\right\rangle \\
& \frac{\Theta_{l}^{(1)}(k, \eta)}{2 l+1}=\int_{0}^{\eta} d \eta^{\prime} e^{-\tau\left(\eta^{\prime}\right)}\left[\dot{\tau}\left(v_{\mathrm{b}}-V\right) j_{l}^{(11)}\left(k\left(\eta-\eta^{\prime}\right)\right)\right. \\
& \left.+\left(\dot{\tau} P^{(1)}\left(k, \eta^{\prime}\right)+\frac{1}{\sqrt{3}} k V\right) j_{l}^{(21)}\left(k\left(\eta-\eta^{\prime}\right)\right)\right], \\
& P^{(1)}(k, \eta)=\frac{1}{10}\left[\Theta_{2}^{(1)}(k, \eta)-\sqrt{6} E_{2}^{(1)}(k, \eta)\right],
\end{aligned}
$$

$$
E_{2}^{(1)}(k, \eta)=-5 \sqrt{6} \int_{0}^{\eta} d \eta^{\prime} e^{-\tau\left(\eta^{\prime}\right)} \dot{\tau} P^{(1)}\left(k, \eta^{\prime}\right) \varepsilon_{2}^{(1)}\left(k\left(\eta-\eta^{\prime}\right)\right),
$$

$$
\begin{aligned}
& j_{l}^{(11)}(x)=\sqrt{\frac{l(l+1)}{2}} \frac{j_{l}(x)}{x}, \\
& j_{l}^{(21)}(x)=\sqrt{\frac{3 l(l+1)}{2}} \frac{d}{d x}\left(\frac{j_{l}(x)}{x}\right),
\end{aligned}
$$

$$
\varepsilon_{2}^{(1)}(x)=\frac{j_{2}(x)}{x^{2}}+\frac{1}{x} \frac{d j_{2}(x)}{d x}
$$

$$
\begin{gathered}
\beta_{l}^{(1)}(x)=\frac{1}{2} \sqrt{(l-1)(l+2)} \frac{j_{l}(x)}{x}, \\
e^{-\tau\left(\eta^{\prime}\right)}=\exp \left(-\int_{\eta^{\prime}}^{\eta} \dot{\tau} d \eta^{\prime \prime}\right),
\end{gathered}
$$

where $j_{l}(x)$ is the spherical Bessel function, $\eta \equiv \int d a / a$ is the conformal time, subscript 0 denotes the present epoch and $\dot{\tau}$ is the differential optical depth which is expressed as

$$
\dot{\tau}=a n_{e} \sigma_{\mathrm{T}}=a / L_{\mathrm{mfp}}
$$

For further notation the reader is referred to Ref. [27]. The conformal time $\eta$ can be written in terms of redshift $z$ as

$$
\eta=\frac{1}{H_{0}} \int \frac{d z}{\sqrt{1-\Omega_{\mathrm{M}}+\Omega_{\mathrm{M}}\left[1+(1+z)\left(1+z_{\mathrm{eq}}^{-1}\right)\right](1+z)^{3}}},
$$

where $z_{\text {eq }}=2.4 \times 10^{4} \Omega_{\mathrm{M}} h^{2}$ is the redshift of the matterradiation equality epoch. In the above expressions, we have taken two independent vector modes into account. The time evolution of $v_{\mathrm{b}}$ is given by the Euler equation.

Combining these formulas with the linear perturbation theory, we can calculate temperature anisotropy and polarization spectra induced by the nonlinear Alfvén modes by simply substituting the fluid velocity of Alfvén modes $v$ in Sec. II to $v_{\mathrm{b}}-V$ of Eq. (65) due to the Newtonian gauge condition [27]. Although, we obtain the Alfvén modes from (fully nonlinear) numerical simulation, we still can treat them as the vector modes because $\nabla \cdot \mathbf{v}=0$.

For the recombination history, we assume the visibility function to be a Gaussian as

$$
\begin{aligned}
g(\eta) & \equiv \dot{\tau} \exp \left(-\int_{\eta}^{\eta_{0}} \dot{\tau} d \eta^{\prime}\right) \\
& =\left(2 \pi \sigma^{2}\right)^{-1 / 2} \exp \left[-\frac{\left(\eta-\eta_{\mathrm{LSS}}\right)^{2}}{\left(2 \sigma^{2}\right)}\right]
\end{aligned}
$$

where $\eta_{\mathrm{LSS}}$ is the conformal time at the LSS and $\sigma$ is the 


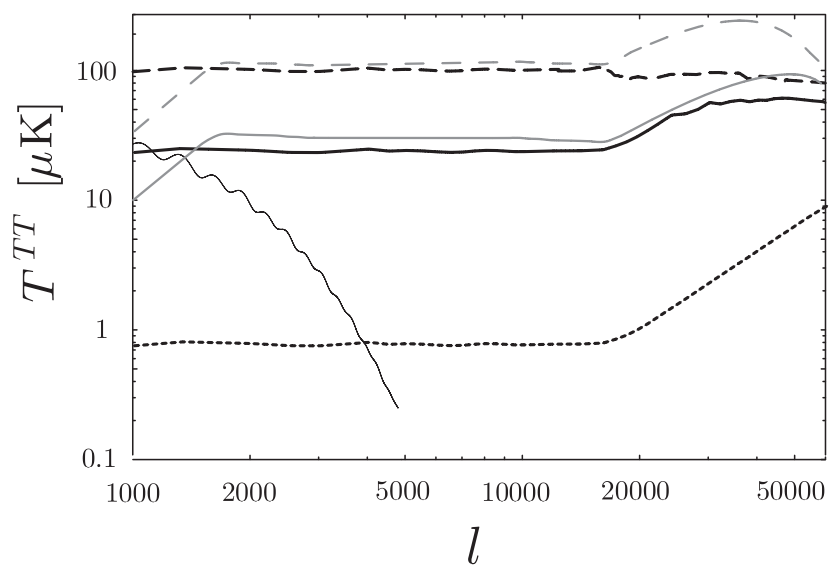

FIG. 4. The temperature anisotropy power spectrum. The dashed line is the case of the magnetic fields with the comoving integral scale $k_{\text {int }}=1.0 \mathrm{Mpc}^{-1}$ with 32 nGauss. The solid line is the case of $k_{\text {int }}=1.5 \mathrm{Mpc}^{-1}$ with 28 nGauss and the dotted line is the case of $k_{\text {int }}=10 \mathrm{Mpc}^{-1}$ with 16 nGauss. The comoving integral scale is related with the magnetic field strength by Eq. (13). The gray line is the linear result of the magnetic field strength 28 nGauss at $1.5 \mathrm{Mpc}$ with spectral index $n=4$. This line has exponential damping at smaller scales than the cutoff scale (for details see Sec. V C). For purpose of comparison, we plot the temperature anisotropy power spectrum in the standard $\lambda$ CDM model (a thin line), computed using CAMB with the same cosmological parameters [28].

width of the LSS. From the WMAP results, the redshift and the thickness of the LSS are $1+z=1089$ and $\Delta z=195$ [23]. This implies that $\sigma$ is $13 \mathrm{Mpc}$ for the cosmological parameters we use in this work.

In our calculations the magnetic field power spectrum stays virtually unchanged after the transition from turbulence to viscous diffusion (i.e. for $t>t_{t-v}$ ), even in the case where $k_{\text {int }}=10 \mathrm{Mpc}^{-1}$ (Case C), since the freestreaming regime before recombination is so short that there is hardly any time for $L_{\text {int }}$ to grow. Since in this study we concentrate on causal spectra $(n>3 / 2)$ we mostly require the magnetic filed spectrum around the integral scale in order to derive the fluid velocity spectrum on all scales. We therefore take the numerical results in the fully developed turbulent regime at $s=2.49$, which is expected to resemble that at $t_{t-v}$ closely. From these numerical spectra of the magnetic fields, we obtain the power spectra of Alfvén modes using Eqs. (44)-(57). We assume the equipartition between magnetic fields and fluid velocities during the turbulent regime, which makes the power spectra flat on large scales (small $l$ 's). The modification of the power spectra due to possible violation of this assumption will be discussed in the next section.

For the calculation of polarization, we compute the source term $P^{(1)}$ by using the publicly available code CAMB $[18,28]$ in which we substitute Alfvén modes obtained by the numerical simulation.

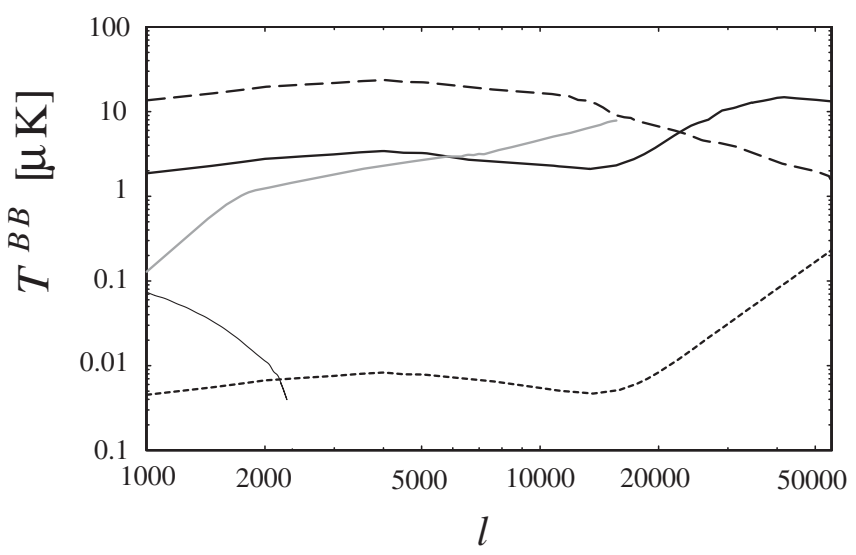

FIG. 5. The B-mode polarization power spectrum. The dashed line is the case of the magnetic fields with the comoving integral scale $k_{\text {int }}=1.0 \mathrm{Mpc}^{-1}$ with $32 \mathrm{nGauss}$ The solid line is the case of $k_{\text {int }}=1.5 \mathrm{Mpc}^{-1}$ with 28 nGauss and the dotted line is the case of $k_{\text {int }}=10 \mathrm{Mpc}^{-1}$ with 16 nGauss. The comoving integral scale is related to the magnetic field strength by Eq. (13). The gray line is the linear result of the magnetic field strength 28 nGauss at $1.5 \mathrm{Mpc}$ with spectral index $n=4$. Because this linear result uses the tight coupling approximation, it is plotted up to the $\sim k_{f} \approx 1.4 \mathrm{Mpc}^{-1}$. For purpose of comparison, we plot the polarization power spectrum in the standard $\lambda \mathrm{CDM}$ model (a thin line), computed using CAMB with the same cosmological parameters. This anisotropies is produced by the gravitational lensing mainly.

Now we are ready to calculate CMB temperature anisotropy and polarization spectra. Hereafter, we fix the initial power law index $n$ to be 4 . Temperature anisotropy and Bmode polarization spectra are shown in Figs. 4 and 5, respectively. Here we calculate models with $k_{\text {int }}=$ $10 \mathrm{Mpc}^{-1}, 1.5 \mathrm{Mpc}^{-1}$ and $1.0 \mathrm{Mpc}^{-1}$ at the LSS, whose magnetic field strengths at the integral scale are 15 nGauss, 28 nGauss, and 32 nGauss, respectively, (see Eq. (13)). Note that $k_{\text {int }}=10 \mathrm{Mpc}^{-1}$ corresponds to Case C, $1.5 \mathrm{Mpc}^{-1}$ to Case $\mathrm{B}$ and $1.0 \mathrm{Mpc}^{-1}$ to Case $\mathrm{A}$ in Sec. III D.

For purpose of comparison, we also plot the temperature anisotropies and polarization by using linear perturbations of the Alfvén modes in Figs. 4 and 5. Here we adopt two types of the magnetic field strength. One is 32 nGauss at $1.0 \mathrm{Mpc}^{-1}$ and another is $28 \mathrm{nGauss}$ at $1.5 \mathrm{Mpc}^{-1}$. In both cases we take the spectral index as $n=4$.

\section{DISCUSSION}

Let us investigate the features of the power spectra of CMB anisotropies and polarization obtained in the previous section. For the qualitative understanding of these spectra, we first develop analytic expressions of CMB anisotropies and polarization.

Both spectra are induced from the power spectrum of the fluid velocity $P_{v}(k)$ at the LSS. In fact, employing the small angle approximation and considering the phase can- 
cellation damping of anisotropies within the thickness of the LSS, we obtain $[16,22]$

$$
\begin{gathered}
\left.\Delta T^{T T}(l) \approx T_{0} \sqrt{\frac{\sqrt{\pi}}{2 k \sigma} \mathcal{P}_{v}(k)}\right|_{k=l /\left(\eta_{0}-\eta_{\mathrm{LSS}}\right)}, \\
\left.\Delta T^{B B}(l) \approx T_{0} \sqrt{\frac{3 \sqrt{\pi}}{2 k \sigma} \mathcal{P}_{P^{(1)}}(k)}\right|_{k=l /\left(\eta_{0}-\eta_{\mathrm{LSS}}\right)},
\end{gathered}
$$

where $\mathcal{P}_{P^{(1)}}(k)$ is the power spectrum of the source term defined as

$$
\mathcal{P}_{P^{(1)}}(k) \equiv \frac{k^{3}}{2 \pi^{2}}\left\langle\left|P^{(1)}\right|^{2}\right\rangle .
$$

Here, $1 / k \sigma$ is due to the phase cancellation damping within the thickness of the LSS.

In Sec. III we studied the velocity power spectra where we found that the shape and amplitude of the power spectra on the LSS depend on the regime of the comoving integral scale at recombination. Note that magnetic fields with the comoving integral scale $k_{\text {int }}=1.2 \mathrm{Mpc}^{-1}$ at recombination are transiting from the viscous regime to the freestreaming regime right at recombination as is shown in Fig. 3. At the recombination epoch, the integral scale is still in the viscous regime for $k_{\text {int }}<1.2 \mathrm{Mpc}^{-1}$, while it has already been in the free-streaming regime for $k_{\text {int }}>$ $1.2 \mathrm{Mpc}^{-1}$.

In the viscous or free-streaming regimes, the velocity power spectra do not explicitly depend on the initial spec- tral index of the magnetic fields $n$ if $n>3 / 2$, which is the case we consider in this paper, as is shown in III B and III C (or summarized in IIID). Even in this initially blue spectrum case, however, the initial spectral index still affects the growth rate of the integral scale (see Eqs. (25) for the turbulent regime and (40) for the free-streaming regime). But as is shown in Fig. 3, the integral scale does not change during the viscous regime and only changes little in the free-streaming regime, provided the magnetic fields are sufficiently strong, i.e. the comoving $B>16 \mathrm{nGauss}$ at recombination. Since the turbulent regime, where the integral scale mostly evolves, is long before the recombination epoch, we can ignore the growth of the integral scale and accordingly the dependence of the initial spectral index. Therefore we expect the velocity power spectra and the resultant temperature anisotropy and polarization spectra to show virtually no dependence on the initial spectral index $n$, for $n>3 / 2$.

\section{A. Temperature anisotropy power spectrum}

Let us now discuss the temperature anisotropies. Following Sec. III D, we investigate the temperature anisotropy spectrum for three separate cases corresponding to the integral scale on the LSS.

First, we consider Case A in which the integral scale $k_{\text {int }}<1.2 \mathrm{Mpc}^{-1}$. The integral scale is still in the viscous regime at the recombination epoch in this case. Substituting the equations of Case A in Sec. IIID into Eq. (76), we can derive the approximations of the temperature anisotropy spectrum produced by the magnetic fields as

$$
\begin{aligned}
& \left.\Delta T^{T T} \approx T_{0} \sqrt{\frac{\sqrt{\pi}}{2 k \sigma}\left(\frac{\left(\rho_{\mathrm{t}}+p_{\mathrm{t}}\right) a}{\nu k_{\mathrm{int}}} \mathcal{P}_{\mathrm{A}}\left(t_{\mathrm{t}-\mathrm{v}}, k_{\mathrm{int}}\right)\right)^{2}\left(\frac{k}{k_{\mathrm{int}}}\right)}\right|_{k=l /\left(\eta_{0}-\eta_{\mathrm{LSs}}\right)} \approx 104\left(\frac{k_{\mathrm{int}}}{1.0 \mathrm{Mpc}^{-1}}\right)^{-13 / 6}[\mu \mathrm{K}], \quad l<l_{\mathrm{int}}, \\
& \left.\Delta T^{T T} \approx T_{0} \sqrt{\frac{\sqrt{\pi}}{2 k \sigma}\left(\frac{\left(\rho_{\mathrm{t}}+p_{\mathrm{t}}\right) a}{\nu k} \mathcal{P}_{\mathrm{A}}\left(t_{\mathrm{t}-\mathrm{v}}, k\right)\right)^{2}}\right|_{k=l /\left(\eta_{0}-\eta_{\mathrm{Lss}}\right)} \approx 104\left(\frac{k_{\mathrm{int}}}{1.0 \mathrm{Mpc}^{-1}}\right)^{-13 / 6}\left(\frac{l}{l_{\mathrm{int}}}\right)^{m-3 / 2}[\mu \mathrm{K}], \quad l_{\mathrm{int}}<l<l_{\mathrm{f}}, \\
& \left.\Delta T^{T T} \approx T_{0} \sqrt{\frac{\sqrt{\pi}}{2 k \sigma}\left(\frac{\left(\rho_{\mathrm{t}}+p_{\mathrm{t}}\right) k}{\rho_{\mathrm{b}} \alpha a} \mathcal{P}_{\mathrm{A}}\left(t_{\mathrm{t}-\mathrm{v}}, k\right)\right)^{2}}\right|_{k=l /\left(\eta_{0}-\eta_{\mathrm{LSs}}\right)} \approx 31.5\left(\frac{k_{\mathrm{int}}}{1.0 \mathrm{Mpc}^{-1}}\right)^{-1 / 6}\left(\frac{l}{l_{\mathrm{int}}}\right)^{m+1 / 2}[\mu \mathrm{K}], \quad l_{\mathrm{f}}<l,
\end{aligned}
$$

where

$$
\begin{gathered}
l_{\text {int }}=k_{\text {int }}\left(\eta_{0}-\eta_{\text {LSS }}\right)=14500\left(\frac{k_{\text {int }}}{1.0 \mathrm{Mpc}^{-1}}\right), \\
l_{\mathrm{f}}=k_{\mathrm{f}}\left(\eta_{0}-\eta_{\text {LSS }}\right)=17800 .
\end{gathered}
$$

We find a very good agreement between these approximations and full numerical calculations as is shown in Fig. 4. On scales $l<l_{\text {int }}$ and for $k \sigma>1$, Eq. (79) suggests no $l$ dependence of the temperature anisotropy spectrum, whereas for $l<l_{\text {int }}$ and $k \sigma<1$, although this region is not shown in Fig. 4, a residual small $l$-dependence is expected to remain $\propto l^{1 / 2}$. Since $m \simeq-2 / 3, l$ dependence on scales $l>l_{\mathrm{f}}$ is also very weak. We expect to see some variation of the temperature anisotropy spectrum between $l_{\text {int }}<l<l_{\mathrm{f}}$. The dashed line in Fig. 4 shows these features.

Note that we assume the equipartition between magnetic fields and fluid velocities during the turbulent regime. On very large scales (small $l$ 's), we may expect violation of this assumption. Above the scale at which this assumption 
is no longer valid, the temperature anisotropy spectrum shows $l$ dependence. In the most extreme case, the advective term can be completely ignored. Therefore the behavior of the power spectrum is described by the linear perturbation as is shown in Appendix ((A29)), and the temperature anisotropy spectrum is proportional to $l^{5 / 2}$. In Fig. 4, this damping on small l's (the blue spectrum) is shown for the linear case. For the nonlinear cases, we do not really know the location of the break from the flat spectrum and the power law index since they strongly depend on when and how the initial magnetic fields were formed. If there existed strong magnetic fields in the very early universe, the assumption of the equipartition is mostly valid, and the flat spectrum on small $l$ 's is expected.

The amplitude of temperature anisotropies in Case A is generally very large. Even in the case when $k_{\text {int }}=$ 1.2 $\mathrm{Mpc}^{-1}$, which corresponds to the smallest amplitude of Case A (or transition between Case A and Case B), $\Delta T^{T T} \simeq 70 \mu \mathrm{K}$. This amplitude is much larger than the one measured by WMAP or BOOMERANG, i.e., $\Delta T^{T T} \simeq$ $40 \mu \mathrm{K}$ at $l \simeq 800$ [29] or $\Delta T^{T T} \simeq 30 \mu \mathrm{K}$ at $l \simeq 1100$ [30], in which major parts of anisotropies should be explained as the primordial anisotropies. Only a small fraction of anisotropies can be interpreted as primordial magnetic field origin at most. Note that we ignore here the possible damping of the temperature spectrum on small l's due to the nonequipartition between magnetic fields and fluid velocities during the turbulent regime. Accordingly the amplitude of temperature anisotropies at $l=800$ and 1100 may be smaller than $70 \mu \mathrm{K}$. Even in the most extreme case with assuming the same power spectrum as the linear calculation, however, we still get $\Delta T^{T T} \simeq 20 \mu \mathrm{K}$ at

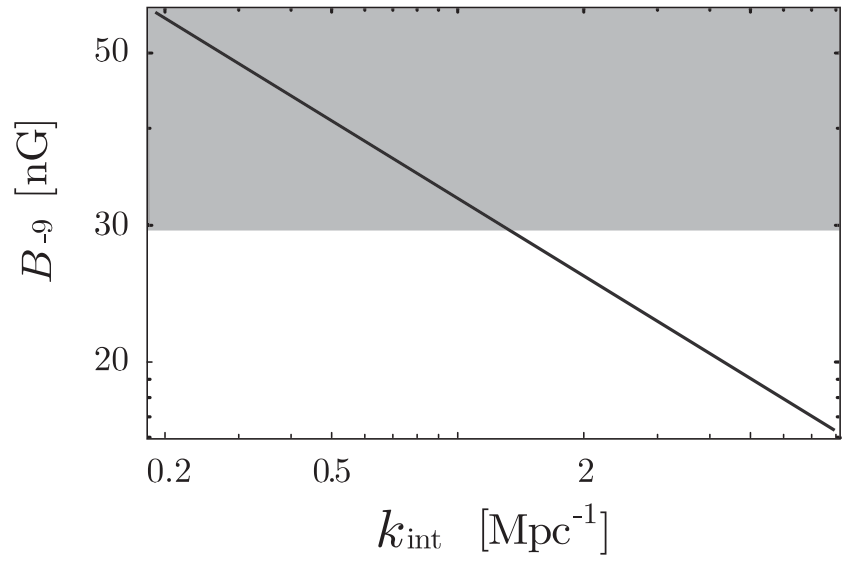

FIG. 6. The magnetic field strength $B_{-9}$ for the comoving integral scale $k_{\text {int }}$ (Eq. (13)). The gray region is the forbidden region by the WMAP results. Magnetic fields with comoving strength larger than 30 nGauss at recombination are ruled out.

$l=1100$, which is about $3 / 2$ of the observed temperature anisotropies. Here we take the break scale $l_{\mathrm{v}}$ is 1800 using the expression in Appendix, and employ the equation $\Delta T^{T T}=70\left(l / l_{\mathrm{v}}\right)^{5 / 2} \mu \mathrm{K}$.

Therefore we can safely rule out the possibility of Case A, i.e., $k_{\text {int }}<1.2 \mathrm{Mpc}^{-1}$ and $B>30$ nGauss at the recombination epoch (see Fig. 6) for $n>3 / 2$.

Now we study Case B. In this case, the integral scale is $1.2 \mathrm{Mpc}^{-1} \lesssim k_{\text {int }} \lesssim 8 \mathrm{Mpc}^{-1}$ and the temperature anisotropies on the LSS are given by the power spectrum in the free-streaming regime. Substituting the equations of Case $\mathrm{B}$ into Eq. (76), we get

$$
\begin{aligned}
& \left.\Delta T^{T T} \approx T_{0} \sqrt{\frac{\sqrt{\pi}}{2 k \sigma}}\left(\frac{1}{\nu} \frac{a}{k_{\mathrm{int}}} \mathcal{P}_{\mathrm{A}}(k)\left(\frac{k}{k_{\mathrm{int}}}\right)^{-n}\right)\left(\frac{k}{k_{\mathrm{int}}}\right)\right|_{k=l /\left(\eta_{0}-\eta_{\mathrm{LSS}}\right)} \approx 43\left(\frac{k_{\mathrm{int}}}{1.5 \mathrm{Mpc}^{-1}}\right)^{-13 / 6}[\mu \mathrm{K}], \quad l<l_{\mathrm{f}}, \\
& \left.\Delta T^{T T} \approx T_{0} \sqrt{\frac{\sqrt{\pi}}{2 k \sigma}\left(\frac{\left(\rho_{\mathrm{t}}+p_{\mathrm{t}}\right) k_{\mathrm{int}}}{\rho_{\mathrm{b}} \alpha a} \mathcal{P}_{\mathrm{A}}\left(t_{\mathrm{t}-\mathrm{v}}, k_{\mathrm{int}}\right)\right)^{2}\left(\frac{k}{k_{\mathrm{int}}}\right)^{5}}\right|_{k=l /\left(\eta_{0}-\eta_{\mathrm{LSS}}\right)} \approx 56\left(\frac{k_{\mathrm{int}}}{1.5 \mathrm{Mpc}^{-1}}\right)^{-1 / 6}\left(\frac{l}{l_{\mathrm{int}}}\right)^{2}[\mu \mathrm{K}], \quad l_{\mathrm{f}}<l<l_{\mathrm{int}}, \\
& \left.\Delta T^{T T} \approx T_{0} \sqrt{\frac{\sqrt{\pi}}{2 k \sigma}\left(\frac{\left(\rho_{\mathrm{t}}+p_{\mathrm{t}}\right) k}{\rho_{\mathrm{b}} \alpha a} \mathcal{P}_{\mathrm{A}}\left(t_{\mathrm{v}-\mathrm{f}}, k\right)\right)^{2}}\right|_{k=l /\left(\eta_{0}-\eta_{\mathrm{LSs}}\right)} \approx 56\left(\frac{k_{\mathrm{int}}}{1.5 \mathrm{Mpc}^{-1}}\right)^{-1 / 6}\left(\frac{l}{l_{\text {int }}}\right)^{m+1 / 2}[\mu \mathrm{K}], \quad l_{\text {int }}<l .
\end{aligned}
$$

Again we find a good agreement between the above approximations and full numerical calculations, i.e., a solid line in Fig. 4. On the scales $l<l_{\mathrm{f}}$ and $l>l_{\text {int }}$, we expect no, or very little $l$ dependence from Eqs. (84) and (86) while the nonequipartition between magnetic fields and fluid velocities may change $l$ dependence in the same manner as Case A. Between $l_{\mathrm{f}}<l<l_{\text {int }}$, however, temperature fluctuations should increase as $l^{2}$. In Fig. 4, the solid line stays flat on the scale $l<18000 \simeq l_{\mathrm{f}}$, and increases until $l \simeq 30000$. However $\Delta T^{T T} \propto l^{2}$ only on the scale $l \lesssim$ $22000 \simeq l_{\text {int }}$ and gradually decreases the gradient for larger $l$.

The WMAP and BOOMERANG results have ruled out Case A. They also constrain Case B as $k_{\text {int }}>1.5 \mathrm{Mpc}^{-1}$, 
or $B<29$ nGauss at the recombination epoch if the equipartition assumption is valid. When the assumption is violated, we cannot set concrete limits for Case B. On the other hand, one might think that CMB anisotropies produced by the magnetic fields can explain the small scale excess of the temperature power spectrum observed by the CBI experiment [31]. However, the power spectrum of CMB anisotropies we find is very flat on scales $2000<l<18000$ even with considering possible violation of the equipartition. Therefore it is rather difficult to explain the CBI experiment which shows Silk damping at $l=2000$ and increases the power to $l=2800$.

Finally let us investigate Case $\mathrm{C}$, in which some further growth of the integral scale takes place before recombination. In this case, the power spectrum Eq. (86) is no longer valid due to the direct cascade while the rests of the power spectra Eq. (84) and (85) are the same. Therefore the difference between Case B and Case C appears in the power spectrum at scales smaller than the integral scale. Employing Eq. (50), we obtain the temperature anisotropy spectrum as

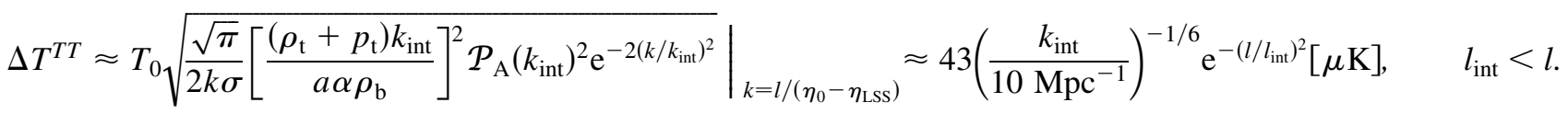

The dotted line of Fig. 4 corresponds to Case C. In this figure, we can find the temperature anisotropy spectrum stays constant for $l<l_{\mathrm{f}}$ and follows $\propto l^{2}$ for $l>l_{\mathrm{f}}$. The integral scale of this model is too large, i.e., $l_{\text {int }}=1.5 \times$ $10^{5}$, to see the difference with Case B.

Note that the temperature anisotropy spectrum induced by the magnetic fields shown in Fig. 4 does not show any significant damping on large $l$ 's (small scales) unlike the primary temperature anisotropy spectrum which suffers Silk damping at $l \simeq 2000$. This is due to the fact that Alfvén modes can survive even below the Silk damping scale [7]. The damping scale for Alfvén modes is the integral scale. Our numerical simulations also show the damping below the integral scale is rather mild compared to the linear calculations. There exists the diffusion scale $v_{\mathrm{A}} / k_{\mathrm{S}}$ below which the power spectrum is exponentially damped for Case C (and Cases A and B in extremely small scales), while we cannot see the damping within the range of Fig. 4. Further comparison with linear calculations will be made in Sec. V C.

\section{B. B-mode polarization power spectrum}

We investigate the B-mode polarization spectrum shown in Fig. 5. For the quantitative understanding, we need to know the behavior of the source term $P^{(1)}$ defined in Eq. (67). In our calculation, we employ CAMB to solve $P^{(1)}$. However, it is rather easy to develop an analytic solution for the evolution of $P^{(1)}$.

First of all, we divide the evolution into two stages. The first one is the period when the tight coupling approximation is valid. Once the wave length of the perturbations becomes shorter than the Silk scale, however, electrons and photons are decoupled and the tight coupling approximation is no longer valid. This gives rise to the second stage.

In the first stage, the solution of the Boltzmann equations of polarization becomes $E_{2}^{(1)}=-(\sqrt{6} / 4) \Theta_{2}^{(1)}$ in the limit of $\tau \rightarrow \infty$ (Eq. (89) of [27]). The quadrupole $(l=2)$ component of the Boltzmann temperature hierarchy gives $\Theta_{2}^{(1)}=(4 \sqrt{3} / 9)(k / \dot{\tau}) \Theta_{1}^{(1)}$. Because of the tight coupling, electron (baryon) velocity follows the photon velocity as $\Theta_{1}^{(1)}=v$. Therefore the source term can be written as (Eq. (94) of [27])

$$
\begin{aligned}
P^{(1)} & \equiv \frac{1}{10}\left[\Theta_{2}^{(1)}-\sqrt{6} E_{2}^{(1)}\right]=\frac{1}{4} \Theta_{2}^{(1)}=\frac{\sqrt{3}}{9} \frac{k}{\dot{\tau}} \Theta_{1}^{(1)} \\
& =\frac{\sqrt{3}}{9} \frac{k L_{\mathrm{mfp}}}{a} v .
\end{aligned}
$$

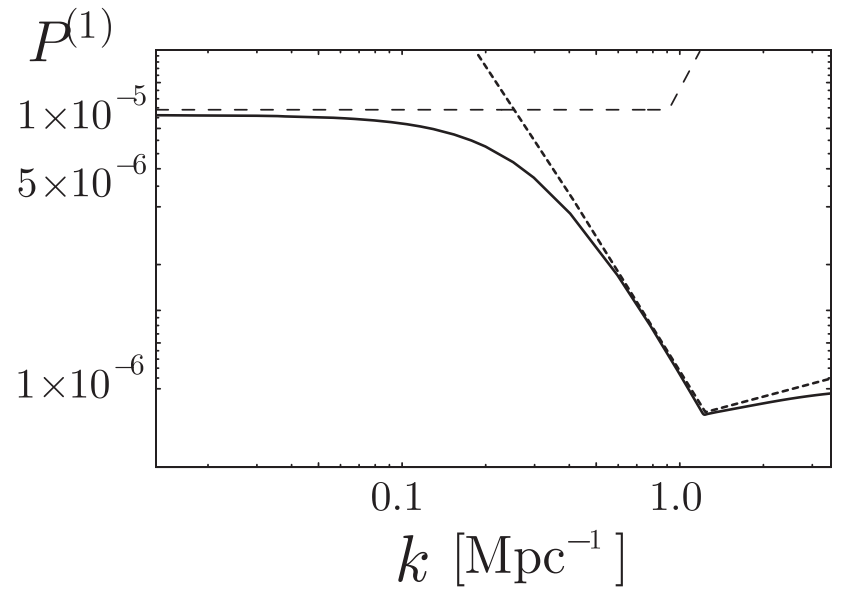

FIG. 7. The Thomson scattering source term $P_{1}(k)$ at the recombination epoch obtained by CAMB for a fixed magnetic field with 1 nGauss amplitude and the scale-invariant power spectrum (solid line). The dashed and dotted lines are the tight coupling approximation, Eq. (88), and the decoupling approximation, Eq. (89), respectively. Note that the Silk damping scale here is $k_{\mathrm{S}} \simeq 0.08 \mathrm{Mpc}^{-1}$, which corresponds to the transition scale between tight coupling and decoupling and the freestreaming scale is $k_{\mathrm{f}} \simeq 1.2 \mathrm{Mpc}^{-1}$. 


\section{HIROYUKI TASHIRO, NAOSHI SUGIYAMA, AND ROBI BANERJEE}

PHYSICAL REVIEW D 73, 023002 (2006)

In the second stage, the E-mode component is damped due to diffusion. Therefore $\Theta_{2}^{(1)}$ induced by the Alfvén modes (Eq. (65)) $v$ only contributes to the source term $P^{(1)}$ in Eq. (67). Namely, $P^{(1)}$ is induced by $v$. It is known that the oscillations of the temperature quadrupole, i.e., $j_{2}^{(11)}(k(\eta-\eta))$ of Eq. (65), suffers damping due to the phase cancellation within the optical depth. Note that the $1 / \sqrt{k} \bar{\sigma}$ coefficient of Eqs. (76) and (77) appeared because of this effect. Therefore we expect to have a factor $1 / \sqrt{k L_{\mathrm{mfp}} / a}$ in the source term $P^{(1)}$. Adding a numerical factor to fit with the simulation, we obtain

$$
P^{(1)} \approx \frac{v}{10 \sqrt{k L_{\mathrm{mfp}} / a}},
$$

which we refer as the decoupling approximation.

For the intuitive understanding, we plot the source term $P^{(1)}\left(k, \eta_{\text {LSS }}\right)$ of the model in which the magnetic fields have a scale-invariant power spectrum, i.e., $k^{3} P_{v}(k) \propto k^{0}$, with a magnetic field strength of nGauss in Fig. 7 by employing CAMB. In this figure, it is found that the numerical calculation follows the tight coupling approximation at scales larger than the Silk damping scale, $k_{\mathrm{S}}=$ $a \sqrt{H / L_{\mathrm{mfp}}} \simeq 0.08 \mathrm{Mpc}^{-1}$. It is also shown that the numerical calculation traces the decoupling approximation Eq. (89) on scales smaller than the Silk damping scale. Gradual increase of the source term on scales $k>k_{\mathrm{f}} \equiv$ $\sqrt{5} a / L_{\mathrm{mfp}} \simeq 1.2 \mathrm{Mpc}^{-1}$ is due to the increase of the velocity $v$ since the viscosity is no longer efficient and the fluid velocity can evolve on these scales (see Eq. (17)).

Now we are ready to calculate the B-mode polarization spectrum using Eq. (77), since the source term $P^{(1)}$ can be written by employing the fluid velocity $v$ as Eqs. (88) and (89). Following our calculations of temperature anisotropies, we obtain a B-mode polarization spectrum for three separate cases, i.e., Cases A, B, and C whose velocity spectra can be seen in Sec. III D.

For Case A, the approximation of the B-mode polarization spectrum is expressed as

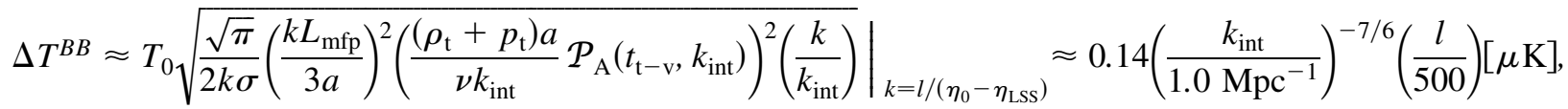

$$
\begin{aligned}
& l<l_{\mathrm{S}} \text {, }
\end{aligned}
$$

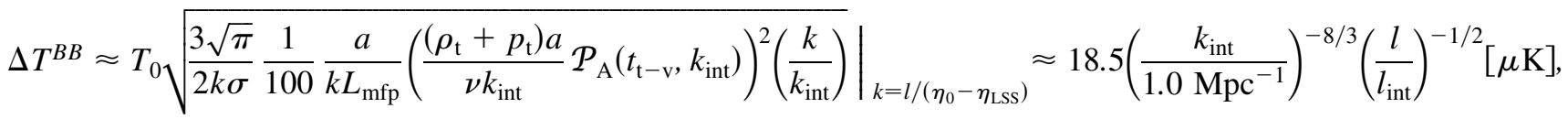

$$
\begin{aligned}
& l_{\mathrm{S}}<l<l_{\text {int }}, \\
& \left.\Delta T^{B B} \approx T_{0} \sqrt{\frac{3 \sqrt{\pi}}{2 k \sigma} \frac{1}{100} \frac{a}{k L_{\mathrm{mfp}}}\left(\frac{\left(\rho_{\mathrm{t}}+p_{\mathrm{t}}\right) a}{\nu k} \mathcal{P}_{\mathrm{A}}\left(t_{\mathrm{t}-\mathrm{v}}, k\right)\right)^{2}}\right|_{k=l /\left(\eta_{0}-\eta_{\mathrm{LSs}}\right)} \approx 18.5\left(\frac{k_{\mathrm{int}}}{1.0 \mathrm{Mpc}^{-1}}\right)^{-8 / 3}\left(\frac{l}{l_{\mathrm{int}}}\right)^{m-2}[\mu \mathrm{K}], \\
& l_{\text {int }}<l<l_{\mathrm{f}}, \\
& \left.\Delta T^{B B} \approx T_{0} \sqrt{\frac{3 \sqrt{\pi}}{2 k \sigma} \frac{1}{100} \frac{a}{k L_{\mathrm{mfp}}}\left(\frac{\left(\rho_{\mathrm{t}}+p_{\mathrm{t}}\right) k}{\rho_{\mathrm{b}} \alpha a} \mathcal{P}_{\mathrm{A}}\left(t_{\mathrm{t}-\mathrm{v}}, k\right)\right)^{2}}\right|_{k=l /\left(\eta_{0}-\eta_{\mathrm{Lss}}\right)} \approx 5.2\left(\frac{k_{\mathrm{int}}}{1.0 \mathrm{Mpc}^{-1}}\right)^{-2 / 3}\left(\frac{l}{l_{\mathrm{f}}}\right)^{m}[\mu \mathrm{K}], \quad l_{f}<l,
\end{aligned}
$$

where

For Case B,

$$
l_{\mathrm{S}}=k_{\mathrm{S}}\left(\eta_{0}-\eta_{\mathrm{LSS}}\right)=1800
$$

$$
\left.\Delta T^{B B} \approx T_{0} \sqrt{\frac{\sqrt{\pi}}{2 k \sigma}\left(\frac{k L_{\mathrm{mfp}}}{3 a}\right)^{2}\left(\frac{\left(\rho_{\mathrm{t}}+p_{\mathrm{t}}\right) a}{\nu k_{\mathrm{int}}} \mathcal{P}_{\mathrm{A}}\left(t_{\mathrm{t}-\mathrm{v}}, k_{\mathrm{int}}\right)\right)^{2}\left(\frac{k}{k_{\mathrm{int}}}\right)}\right|_{k=l /\left(\eta_{0}-\eta_{\mathrm{LSS}}\right)} \approx 0.09\left(\frac{k_{\mathrm{int}}}{1.5 \mathrm{Mpc}^{-1}}\right)^{-7 / 6}\left(\frac{l}{500}\right)[\mu \mathrm{K}], \quad l<l_{\mathrm{S}},
$$

$$
\begin{aligned}
\Delta T^{B B} & \left.\approx T_{0} \sqrt{\frac{3 \sqrt{\pi}}{2 k \sigma} \frac{1}{100} \frac{a}{k L_{\mathrm{mfp}}}\left(\frac{\left(\rho_{\mathrm{t}}+p_{\mathrm{t}}\right) a}{\nu k_{\mathrm{int}}} \mathcal{P}_{\mathrm{A}}\left(t_{\mathrm{t}-\mathrm{v}}, k_{\mathrm{int}}\right)\right)^{2}\left(\frac{k}{k_{\mathrm{int}}}\right)}\right|_{k=l /\left(\eta_{0}-\eta_{\mathrm{LSS}}\right)} \approx 26\left(\frac{k_{\mathrm{int}}}{1.5 \mathrm{Mpc}^{-1}}\right)^{-8 / 3}\left(\frac{l}{l_{\mathrm{f}}}\right)^{-1 / 2}[\mu \mathrm{K}], \\
l_{\mathrm{S}} & <l<l_{\mathrm{f}},
\end{aligned}
$$




$$
\begin{aligned}
\Delta T^{B B} & \left.\approx T_{0} \sqrt{\frac{3 \sqrt{\pi}}{2 k \sigma} \frac{1}{100} \frac{a}{k L_{\mathrm{mfp}}}\left(\frac{\left(\rho_{\mathrm{t}}+p_{\mathrm{t}}\right) k_{\mathrm{int}}}{\rho_{\mathrm{b}} \alpha a} \mathcal{P}_{\mathrm{A}}\left(t_{\mathrm{t}-\mathrm{v}}, k_{\mathrm{int}}\right)\right)^{2}\left(\frac{k}{k_{\mathrm{int}}}\right)^{5}}\right|_{k=l /\left(\eta_{0}-\eta_{\mathrm{Lss}}\right)} \approx 9.9\left(\frac{k_{\mathrm{int}}}{1.5 \mathrm{Mpc}^{-1}}\right)^{-2 / 3}\left(\frac{l}{l_{\mathrm{int}}}\right)^{3 / 2}[\mu K], \\
l_{\mathrm{f}} & <l<l_{\mathrm{int}},
\end{aligned}
$$

$$
\left.\Delta T^{B B} \approx T_{0} \sqrt{\frac{3 \sqrt{\pi}}{2 k \sigma} \frac{1}{100} \frac{a}{k L_{\mathrm{mfp}}}\left(\frac{\left(\rho_{\mathrm{t}}+p_{\mathrm{t}}\right) k}{\rho_{\mathrm{b}} \alpha a} \mathcal{P}_{\mathrm{A}}\left(t_{\mathrm{v}-\mathrm{f}}, k\right)\right)^{2}}\right|_{l=k\left(\eta_{0}-\eta_{\mathrm{Lss}}\right)} \approx 9.9\left(\frac{k_{\mathrm{int}}}{1.5 \mathrm{Mpc}^{-1}}\right)^{-2 / 3}\left(\frac{l}{l_{\mathrm{int}}}\right)^{m}, \quad l_{\mathrm{int}}<l .
$$

Finally, for Case C, the difference with Case B only arises at the scales smaller than the integral scale as is the case of temperature anisotropies. Using Eq. (50), we acquire the B-mode polarization spectrum as

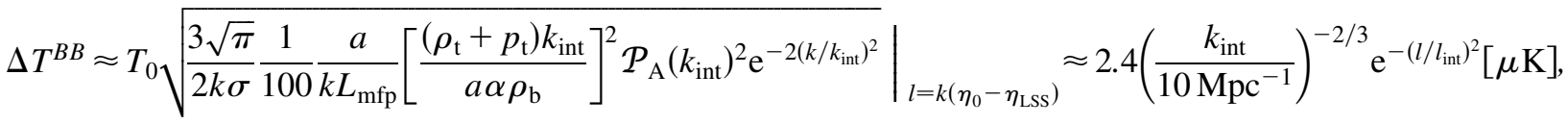

$$
\begin{aligned}
& l_{\text {int }}<l \text {. }
\end{aligned}
$$

Let us discuss our numerical results of B-mode power spectra in Fig. 5. The dashed, solid and dotted lines correspond to Cases $\mathrm{A}, \mathrm{B}$, and $\mathrm{C}$. It is shown that all lines gradually increase until $l \sim 4000$, since $\Delta T^{B B} \propto l$ on scales $l<l_{\mathrm{S}}$ for all cases. Note that we assume the equipartition between magnetic fields and fluid velocities during the turbulent regime. On very large scales (small $l$ 's), we may expect violation of this assumption and steeper slopes for the B-mode power spectra below $l \lesssim 2000$ as is the case with temperature spectra. Case A shows continuous declining on $l>4000$ which is consistent with our analytic estimate. The increase of $\Delta T^{B B}$ on scales $l>l_{\mathrm{f}}=$ 17800 for Cases B and C is caused by the efficient evolution of the velocity field in the free-streaming regime due to the lack of dissipation term $\mathbf{f}$. For both Cases B and C, $\Delta T^{B B}$ decreases on scales $l>l_{\text {int }}$. We can see this decrease in Fig. 5 for Case B whose $l_{\text {int }}$ is 22000 . For Case C, we cannot find this trend because $l_{\text {int }}=1.4 \times 10^{5}$, which is beyond the range of Fig. 5 .

From the temperature anisotropy spectrum, Case A and a part of Case B are excluded. However, the solid line in Fig. 5, which overshoots the B-mode produced by the gravitational lens effect, is not yet ruled out. Even the dotted line can provide dominant contribution as the Bmode polarization on scales $l>3000$.

\section{Comparison with linear calculations}

Here, we compare our results with linear calculations. Note that all previous works investigated the effects of the magnetic fields on CMB anisotropies and polarization are based on linear perturbation theory.

In the Appendix, we summarize the linear perturbation analysis and the approximated estimations of temperature anisotropy and polarization spectra following the analysis by Subramanian and Barrow ([16,22]).

In Fig. 4, we plot the temperature power spectrum of the linear calculation with $B=28 \mathrm{nGauss}$ at $k=1.5 \mathrm{Mpc}^{-1}$ and $n=4$ as the gray line for comparison. It is shown that differences with the nonlinear calculation (the solid line) appear on scales $l<2000$, and $l>18000$.

On large scales, we can ignore the diffusion in the early epoch. In the nonlinear calculation, $v$ immediately approaches the Alfvén velocity $v=v_{\mathrm{A}}$ due to the nonlinear coupling during the turbulent regime if there exist strong magnetic fields in the very early universe. On the other hand, the growth of the velocity induced by the magnetic pressure gradient in the linear calculation turns out to be much slower as is shown in the Appendix (Eq. (A9)). Accordingly we find a smaller temperature power spectrum (and polarization) for the linear calculation. Note that even for the nonlinear calculation, there may be the case in which the equipartition could not be achieved by the end of the turbulent regime if the primordial magnetic fields on the large scale were not strong enough. In this case, we rather expect to have similar behaviors of the power spectrum to the linear one on large scales (small $l$ 's).

Once the viscosity becomes efficient, the velocity follows the linear solution even in the case of the nonlinear calculation (see Eqs. (15) and (A10)). Unlike the perturbations without the magnetic fields which suffer severe damping, the velocity (Alfvén mode) induced by the magnetic fields can survive within the Silk scale for both nonlinear and linear calculations. The amplitude of the velocity is determined by the Alfvén velocity, i.e., the amplitude of the magnetic fields. Therefore both linear and nonlinear calculations give almost identical results, which are $\Delta T^{T T}(l) \propto l^{0}$, between $2000\left(\sim l_{\mathrm{S}}\right)<l<18000$.

On small scales, $l>18000$, the linear calculation shows steeper rise of the power spectra for both Cases A and B than the nonlinear calculation as shown in Fig. 4. There also exists exponential damping in the linear calculation on smaller scales. On the other hand, nonlinear calculation shows less rise and no damping in the power spectra.

The reason why the power spectra of the nonlinear calculation have less prominent peaks on the small scales (large $l$ 's) is due to the cascade decay of the magnetic fields 
during the turbulent regime. As shown in Fig. 2, the peak of the magnetic field power spectrum shifts to larger scales. The condition of this shift is determined by $t_{\text {eddy }} \equiv$ $L_{\text {int }} / v_{\mathrm{A}}=1 / H$ as is discussed in Sec. II A. Then the cutoff scale (or integral scale) has been frozen since the transition from the turbulent regime to the viscus regime. Accordingly the comoving wave number of the cutoff can be written and $k_{\text {int }}=a H /\left.v_{\mathrm{A}}\right|_{z_{t-v}}$ as is obtained in Eq. (13). On the other hand, the cutoff scale of the linear calculation is always determined by the diffusion condition $k_{\mathrm{c}}=k_{\mathrm{S}} / v_{\mathrm{A}}$ (see Appendix). The power spectrum increases toward small scales as long as $k<k_{\mathrm{c}}$. Then it starts to show exponential damping at $k_{\mathrm{c}}$. In Cases $\mathrm{A}$ and $\mathrm{B}$, $k_{\text {int }}<k_{\mathrm{c}}$ at the recombination epoch. Therefore nonlinear calculation shows less peaks in the power spectra for these cases. The diffusion damping scale is determined by $k_{\mathrm{c}}=$ $k_{\mathrm{S}} / v_{\mathrm{A}} \propto 1 / B$ for both linear and nonlinear calculations. Therefore one might expect to have same damping behaviors for both linear and nonlinear calculations. However, since the magnetic field strength of the nonlinear calculation on the scales smaller than $1 / k_{\text {int }}$ is smaller than the one of the linear calculation as well as temperature anisotropies, we expect to have the smaller diffusion damping scale for the nonlinear calculation than the linear calculation, i.e., $k_{\mathrm{c}}^{\text {nonlinear }}>k_{\mathrm{c}}^{\text {linear }}$. Within the range of Fig. 4 , we can only see the diffusion (exponential) damping of the linear calculation. The nonlinear calculations for Cases A and B only show much milder damping due to the cascade decay during the turbulent regime below the integral scale.

In Case $\mathrm{C}, k_{\text {int }}>k_{\mathrm{c}}$ at the recombination epoch. Therefore we ought to obtain very similar temperature power spectra for both linear and nonlinear calculations.

\section{CONCLUSION}

In this paper we study the effect of the primordial magnetic fields on the CMB temperature and polarization anisotropies. In particular the nonlinear evolution of the magnetic fields and the resulting Alfvén modes of the fluid velocities, which are the source of temperature anisotropies and polarization, are appropriately included based on the cosmological MHD simulation by Banerjee and Jedamzik $[10,11]$. Diffusion and direct cascade processes are properly taken into account.

We separate the evolution into three regimes, i.e., turbulent, diffusion, and free-streaming. In the turbulent regime, the advective term, which is essentially nonlinear, provides the dominant contribution on the evolution of Alfvén modes. Viscosity plays an important role in the viscous regime, while the drag term takes over the task of the viscous term in the free-streaming regime.

By combining the numerical simulations of three regimes, we obtain a comprehensive evolution history of the magnetic fields and Alfvén modes. We find the relation between the integral (or coherent) scale on which the magnetic energy peaks, and the maximum magnetic field strength, or equivalently, the magnetic field strength at the integral scale as shown in Fig. 6.

We divide the evolution of the magnetic fields and the Alfvén modes into three cases, i.e., Cases A, B, and C. For Case $\mathrm{A}$, the integral scale is still in the viscous regime at the recombination epoch, while the integral scale is in the free-streaming regime for Cases $\mathrm{B}$ and $\mathrm{C}$. The direct cascade process takes place during the turbulent regime for all cases. In the free-streaming regime the direct cascade process as well as the diffusion process works for Case $\mathrm{C}$ at the integral scale. The resultant velocity spectra of Alfvén modes are quite different between three cases.

From the velocity spectra, we calculate CMB temperature anisotropy and (B-mode) polarization spectra. We make comparisons between nonlinear and linear calculations and find differences on both large and small scales. On large scales $l<2000$, both CMB anisotropy and polarization spectra have flat and blue spectra for the nonlinear and linear calculations, respectively. This difference is caused by the inclusion of the advective term for the nonlinear calculations. This difference gives stronger constraints for the nonlinear case in the intermediate angular scale using CMB observations such as WMAP and BOOMERANG. Note that the possible nonequipartition in the turbulent regime may make the difference between nonlinear and linear calculations small.

Using WMAP and BOOMERANG, we set rough upper limits for the comoving magnetic field strength as $B<$ 28 nGauss and the comoving integral scale as $k_{\text {int }}>$ $1.5 \mathrm{Mpc}^{-1}$ if the equipartition is valid in the turbulent regime. If this assumption is violated, we still can set rough upper limits as $B<30 \mathrm{nGauss}$ and $k_{\text {int }}>1.2 \mathrm{Mpc}^{-1}$.

For the polarization spectra, we also expect higher signals on large scales by nonlinear calculations than linear ones. The signal may exceed the B-mode polarization from the gravitational lenses if primordial magnetic fields exist.

On small scales, nonlinear calculations show milder damping of temperature anisotropies and polarization than linear calculations in Cases A and B. We expect to have both temperature anisotropy and polarization spectra even beyond $l>10000$. The peak values of temperature anisotropy and B-mode polarization spectra are approximately $40 \mu \mathrm{K}$ and a few $\mu \mathrm{K}$ depending on the peak scale $l_{\text {int }}$ (or the integral scale $k_{\text {int }}$ ).

Note that we consider only the case with the spectral index of the magnetic field spectrum $n>3 / 2$ in this paper, while the extension to the case with $0<n<3 / 2$ is straightforward.

Various observation projects for the small scale temperature anisotropies and polarization are planed [32]. These observations may find the evidence of primordial magnetic fields or at least will set stringent constraints. Perhaps the best constraint will be provided by the really fine scale data, i.e., $l>10000$.

Finally, another and possibly stronger constraint on the field strength of putative primordial magnetic fields might 
be derived from the deflection of ultra high energy cosmic rays (UHECR). Dolag et al. [33] simulated the evolution of magnetic fields using a MHD SPH code and solved the propagation of UHECR. Their seed magnetic fields were only $2 \times 10^{-12}$ Gauss and they found that strong deflections of UHECR with arrival energies $E=4 \times 10^{19}$ within a distance of $100 \mathrm{Mpc}$ occur only when UHECR cross galaxy clusters. Therefore the arrival directions of UHECR mostly trace the source positions on the sky. On the other hand, if there exists the magnetic fields of 10 nGauss as a seed, we expect to have very strong deflections for UHECR with arrival energies even above $10^{20} \mathrm{eV}$. In near future, a huge amount of UHECR will be observed by the air shower experiments such as the Pierre Auger observatory [34] whose angular resolution is $\sim 0.6$ degree [35]. If the observed UHECR with $10^{20} \mathrm{eV}$, which only can arrive from nearby sources ( $\lesssim 100 \mathrm{Mpc}$ ) due to GZK cutoff [36], do trace the large scale structure, we will be able to set a very stringent upper limit for the primordial magnetic field strength. Note that the propagation and the deflection of UHECR are still open questions. Contrary to Dolag et al., Sigl et al. found larger deflection of UHECR in their simulation [37]. They claimed that the sources are strongly magnetized $(\sim \mu$ Gauss) and the deflection angle can be of order $20^{\circ}$ up to $10^{20} \mathrm{eV}$ even if the extragalactic magnetic fields of the observer are negligible ( $\ll 0.1 \mu$ Gauss). If this is the case, however, we can still set an upper limit to the magnetic filed strength, if the observed UHECR above $10^{20} \mathrm{eV}$ do trace the large scale structure. In near future, a huge amount of UHECR will be observed by the air shower experiments such as the Pierre Auger observatory [34] whose angular resolution is $\sim 0.6$ degree [35]. If the observed UHECR with $10^{20} \mathrm{eV}$, which only can arrive from nearby sources ( $\lesssim 100 \mathrm{Mpc}$ ) due to GZK cutoff [36], do trace the large scale structure, we will be able to set a very stringent upper limit for the primordial magnetic field strength.

\section{ACKNOWLEDGMENTS}

We would like to thank Karsten Jedamzik for many crucial comments. One of the authors (N.S.) would like to thank Wayne Hu for useful comments and also thank for kind hospitality of Kavli institute for Cosmological Physics at the University of Chicago. N.S. is supported by a Grant-in-Aid for Scientific Research from the Japanese Ministry of Education (No. 17540276).

\section{APPENDIX: LINEAR PERTURBATION}

In this appendix, we review the linear perturbation analysis of the magnetic fields in the early universe. For a detailed discussion, see the references [16,17,22].

We assume that the metric perturbations of the vector mode are expressed as Eq. (58). The stress energy tensors are divided into the fluid-part, $T_{\mathrm{F}}^{\mu \nu}$, and the magnetic field part, $T_{\mathrm{B}}^{\mu \nu}$. In the fluid part, we take into account of the viscosity between photons and baryons (electrons)

$$
\begin{gathered}
T_{\mathrm{F}}^{\mu \nu}=\left(p_{\mathrm{t}}+\rho_{\mathrm{t}}\right) U^{\mu} U^{\nu}+p_{\mathrm{t}} g^{\mu \nu}-\eta H^{\mu \alpha} H^{\nu \beta} W_{\alpha \beta}, \\
H^{\alpha \beta} \equiv g^{\alpha \beta}+U^{\alpha} U^{\beta}, \\
W_{\alpha, \beta} \equiv U_{\alpha ; \beta}+U_{\beta ; \alpha}-\frac{2}{3} g_{\alpha \beta} U_{; \gamma}^{\gamma}, \quad \eta=\frac{4}{15} \rho_{\gamma} L_{\mathrm{mfp}} .
\end{gathered}
$$

Here $U^{\mu}$ is the four-velocity and is written as

$$
U^{0}=1 / a, \quad U^{i}=u^{i} / a,
$$

where $u^{i}$ are velocities. Now we are interested in the vector mode of the stress energy tensor perturbations, so that the velocity filed is divergence free and is decomposed as Eq. (60).

The magnetic part of the stress energy tensor is expressed as

$$
\begin{aligned}
T_{\mathrm{B} i j}(\mathbf{k})= & \frac{1}{4 \pi} \int d^{3} p\left[B_{i}(\mathbf{p}) B_{j}(\mathbf{k}-\mathbf{p})\right. \\
& \left.-\frac{1}{2} \delta_{i j} B_{l}(\mathbf{p}) B_{l}(\mathbf{k}-\mathbf{p})\right] .
\end{aligned}
$$

For obtaining the vector mode of the magnetic part, we introduce a projection tensor onto the transverse plane as

$$
P_{i j}(\mathbf{k}) \equiv \delta_{i j}-k_{i} k_{j} / k^{2} .
$$

Then the vector mode of Eq. (A4) can be written as

$$
T_{\mathrm{B} i j}^{V}=\left(P_{i n} k_{j}+P_{j n} k_{i}\right) k_{m} T_{B m n} / k^{2} .
$$

From the conservation law of the energy momentum tensors $T_{F}$ and $T_{B}$, we obtain the Euler equation

$$
\begin{gathered}
\left(\dot{u}_{i}-\dot{V}_{i}\right)+\left(1-3 c_{\mathrm{st}}\right) \frac{\dot{a}}{a}\left(u_{i}-V_{i}\right)+\frac{\nu}{a} \frac{k^{2}}{\rho_{\mathrm{t}}+p_{\mathrm{t}}}\left(u_{i}-V_{i}\right) \\
=\frac{k \Pi_{i}}{a^{4}\left(\rho_{\mathrm{t}}+p_{\mathrm{t}}\right)},
\end{gathered}
$$

where dots represent the derivatives with respect to the conformal time and the comoving pressure gradient term of the magnetic field $\Pi_{i}$ is defined as

$$
\frac{\Pi_{i}}{a^{4}}=P_{i n} \hat{k}_{m} T_{\mathrm{B}}^{m n} .
$$

As discussed in Sec. IV, we use $v_{i}$ which is defined as $v_{i} \equiv u_{i}-V_{i}$. We can rewrite Eq. (A7) in terms of $v_{i}$. When we evaluate Eq. (A7) at large scales where the viscosity can be neglected as is the case of the turbulent regime in the nonlinear calculations, we obtain as the following approximation in the radiation dominated epoch

$$
v_{i}=\frac{3 k \Pi_{i} \eta}{4(R+1) \rho_{\gamma} a^{4}},
$$


where $R=3 \rho_{\mathrm{b}} / 4 \rho_{\gamma}$. We refer the scales where Eq. (A9) is valid as "no diffusion scales".

At small scales where the viscosity is dominant over the cosmological expansion, which we call as "viscous scales", we can neglect the expansion term in Eq. (A7) and obtain

$$
v_{i}=\frac{15 \Pi_{i}}{4 \rho_{\gamma} a^{3} k L_{\mathrm{mfp}}},
$$

with employing the terminal-velocity approximation.

Matching Eqs. (A9) and (A10), we acquire the transition scale between the no diffusion scales and the viscous scales as

$$
k_{\mathrm{v}} \sim\left[\frac{5 a(1+R)}{\eta L_{\mathrm{mfp}}}\right]^{1 / 2} .
$$

At the smaller scales than the photon mean free path, which we refer as "free-streaming scales", baryons and photons are decoupled and we can no longer adopt the diffusion approximation, Eq. (A7). Instead, we must introduce the drag force term $\dot{\tau} v_{i} / R$ in the baryon Euler equation as

$$
\dot{v}_{i}+\frac{\dot{a}}{a} v_{i}+\frac{\dot{\tau}}{R} v_{i}=\frac{k \Pi_{i}}{\rho_{\mathrm{b}} a^{4}} .
$$

When we neglect the cosmological expansion term and apply the terminal-velocity approximation we obtain

$$
v_{i}=\frac{3 k \Pi_{i} L_{\mathrm{mfp}}}{4 a^{5} \rho_{\gamma}}
$$

The transition scale between the viscous scales and the free-streaming scales is $k_{\mathrm{f}}$ as discussed in Sec. III B because Eqs. (A10) and (A13) are identical to Eqs. (15) and (17).

For the comparison with the nonlinear results we calculate the power spectrum of the velocity fields. From the above results $v_{i}$ is rewritten as

$$
\begin{gathered}
v_{i}=k X(\eta) \Pi_{i}, \\
X(\eta)= \begin{cases}3 \tau / 4 \rho_{\gamma} a^{4}(1+R), & k<k_{\mathrm{v}}, \\
15 / 4 \rho_{\gamma} a^{3} k^{2} L_{\mathrm{mfp}}, & k_{\mathrm{v}}<k<k_{\mathrm{f}}, \\
3 L_{\mathrm{mfp}} / 4 \rho_{\gamma} a^{5}, & k_{\mathrm{f}}<k .\end{cases}
\end{gathered}
$$

The ensemble average of the velocity fields leads to

$$
\left\langle\left|v_{i}\right|^{2}\right\rangle=k^{2} X(\eta)^{2}\left\langle\left|\Pi_{i}\right|^{2}\right\rangle .
$$

By following the procedure of Ref. [17], $\left\langle\left|\Pi_{i}\right|^{2}\right\rangle$ can be solved as

$$
\begin{aligned}
\left\langle\left|\Pi_{i}\right|^{2}\right\rangle= & \frac{1}{(4 \pi)^{2}} \int_{0}^{\infty} d q^{3} \frac{\mathcal{P}_{B}(q)}{q^{3}} \frac{\mathcal{P}_{B}(|(\mathbf{k}+\mathbf{q})|)}{|(\mathbf{k}+\mathbf{q})|^{3}}\left(1-\mu^{2}\right) \\
& \times\left[1+\frac{(k+2 q \mu)(k+q \mu)}{\left(k^{2}+q^{2}+2 k q \mu\right)}\right],
\end{aligned}
$$

where $q=|\mathbf{q}|$ and $\mu=\mathbf{k} \cdot \mathbf{q} /(q k)$. Now, we assume that the magnetic power spectrum is the power law spectrum with the spectral index $n$ as

$$
\mathcal{P}_{B}=\frac{k^{3}}{2 \pi^{2}}\left\langle\left|B_{\text {comov }}\right|^{2}\right\rangle=B_{-9}^{2}\left(\frac{k}{k_{\mathrm{n}}}\right)^{n},
$$

where the strength of the magnetic fields is $B_{-9}$ nGauss at the wave length $k_{\mathrm{n}}$.

Assuming the power law spectrum for the magnetic fields, we can obtain the analytic approximation of $\left\langle\left|\Pi_{i}\right|^{2}\right\rangle[16,17,22]$. Accordingly, the power spectrum of the fluid velocity leads to

$$
\mathcal{P}_{v}(k)=\frac{k^{3}}{2 \pi^{2}}\left\langle\left|v_{i}\right|^{2}\right\rangle \approx k^{2} B_{-9}^{2} X(\eta)^{2} I(k)^{2},
$$

where the mode coupling $I(k)$ is different for the spectrum with the index $n<3 / 2$ and $n>3 / 2$. From the reference [16], in the case of $n<3 / 2$, the mode coupling $I(k)$ is approximated as

$$
I^{2}(k)=\frac{1}{32 \pi^{4}} \frac{2}{3 n}\left(\frac{k}{k_{\mathrm{n}}}\right)^{2 n} .
$$

In the case of $n>3 / 2$,

$$
I^{2}(k)=\frac{1}{32 \pi^{4}} \frac{7}{15(2 n-3)}\left(\frac{k}{k_{\mathrm{n}}}\right)^{3}\left(\frac{k_{\mathrm{c}}}{k_{\mathrm{n}}}\right)^{2 n-3},
$$

where $k_{\mathrm{c}}$ is the cutoff scale defined as $k_{\mathrm{c}} \equiv k_{\mathrm{S}} / v_{\mathrm{A}}[7,8]$. This cutoff of the power spectrum is caused by the dissipation due to the drag force.

For the comparison with the nonlinear results in this paper, we focus on the case with the blue spectrum $n>$ $3 / 2$. Using Eq. (A21), we obtain the power spectrum of the fluid velocity as

$$
\mathcal{P}_{v}(k) \approx k^{2}\left[\frac{3 \eta}{4 \rho_{\gamma} a^{4}(1+R)}\right]^{2} I(k)^{2} \propto k^{5}, \quad k<k_{\mathrm{v}},
$$

$$
\mathcal{P}_{v}(k) \approx k^{2}\left[\frac{15}{4 \rho_{\gamma} a^{3} k^{2} L_{\mathrm{mfp}}}\right]^{2} I(k)^{2} \propto k, \quad k_{\mathrm{v}}<k<k_{\mathrm{f}},
$$

$$
\mathcal{P}_{v}(k) \approx k^{2}\left[\frac{3 L_{\mathrm{mfp}}}{4 \rho_{\gamma} a^{5}}\right]^{2} I(k)^{2} \propto k^{5}, \quad k_{\mathrm{f}}<k .
$$

From the velocity power spectrum, CMB temperature anisotropy and polarization spectra can be calculated. Subramanian and Barrow [16,22] obtained the following results with using the small angle approximation.

In the case of $k \sigma \ll 1$,

$$
\Delta T^{T T}=\left.T_{0} \sqrt{\frac{l(l+1) C(l)}{2 \pi}} \approx T_{0} \sqrt{\frac{\pi}{2} \mathcal{P}_{v}(k)}\right|_{k=l /\left(\eta_{0}-\eta_{\mathrm{LSS}}\right)},
$$




$$
\begin{aligned}
\Delta T^{B B} & =T_{0} \sqrt{\frac{l(l+1) C(l)}{2 \pi}} \\
& \left.\approx T_{0} \sqrt{\frac{\pi}{2}\left(\frac{k L_{\mathrm{mfp}}}{3 a}\right)^{2} P_{v}(k)}\right|_{k=l /\left(\eta_{0}-\eta_{\mathrm{LSS}}\right)} .
\end{aligned}
$$

And for the case of $k \sigma \gg 1$,

$$
\begin{aligned}
\Delta T^{T T}= & \left.T_{0} \sqrt{\frac{l(l+1) C(l)}{2 \pi}} \approx T_{0} \sqrt{\frac{\pi^{1 / 2}}{2 k \sigma} P_{v}(k)}\right|_{k=l /\left(\eta_{0}-\eta_{\mathrm{LSS}}\right)}, \\
\Delta T^{B B} & =T_{0} \sqrt{\frac{l(l+1) C(l)}{2 \pi}} \\
& \left.\approx T_{0} \sqrt{\frac{\pi^{1 / 2}}{2 k \sigma}\left(\frac{k L_{\mathrm{mfp}}}{3 a}\right)^{2} P_{v}(k)}\right|_{k=l /\left(\eta_{0}-\eta_{\mathrm{LSS}}\right)} . \quad \text { (A28) }
\end{aligned}
$$

The temperature anisotropy spectrum induced by the magnetic fields with spectral index $n>3 / 2$ in the low multipoles is given by substituting Eq. (A22) into Eq. (A25) and the one in the high multipoles is given by Eqs. (A23) and (A24) into Eq. (A27) as

$$
\Delta T^{T T} \approx 5.4 B_{-9}^{2}\left(\frac{l}{1000}\right) I\left(k_{l}\right)[\mu \mathrm{K}] \propto l^{5 / 2}, \quad l<l_{\mathrm{v}},
$$

$$
\begin{aligned}
\Delta T^{T T} & \approx 13.0 B_{-9}^{2}\left(\frac{l}{2000}\right)^{-3 / 2} I\left(k_{l}\right)[\mu \mathrm{K}] \propto k^{0}, \\
l_{\mathrm{v}} & <l<l_{\mathrm{f}},
\end{aligned}
$$

$$
\begin{gathered}
\Delta T^{T T} \approx 0.4 B_{-9}^{2}\left(\frac{l}{20000}\right)^{1 / 2} I\left(k_{l}\right)[\mu \mathrm{K}] \propto l^{2}, \\
l_{\mathrm{f}}<l<l_{\mathrm{c}}, \\
\quad I\left(k_{l}\right)=\left.I(k)\right|_{k=l /\left(\eta_{0}-\eta_{\mathrm{LSS}}\right)}
\end{gathered}
$$

where $l_{\mathrm{v}}=k_{\mathrm{v}}\left(\eta_{0}-\eta_{\mathrm{LSS}}\right)$ and $l_{\mathrm{c}}=k_{\mathrm{c}}\left(\eta_{0}-\eta_{\mathrm{LSS}}\right)$.

The B-mode polarization spectrum induced by the magnetic fields with spectral index $n>3 / 2$ is acquired by substituting Eq. (A22) into Eq. (A26) and substituting Eq. (A23) into Eq. (A28) as

$$
\Delta T^{B B} \approx 0.04 B_{-9}^{2}\left(\frac{l}{1000}\right)^{2} I\left(k_{l}\right)[\mu \mathrm{K}] \propto l^{7 / 2}, \quad l<l_{\mathrm{v}},
$$

$$
\begin{aligned}
\Delta T^{B B} & \approx 0.12 B_{-9}^{2}\left(\frac{l}{2000}\right)^{-1 / 2} I\left(k_{l}\right)[\mu \mathrm{K}] \propto l, \\
l_{\mathrm{v}} & <l<l_{\mathrm{f}} .
\end{aligned}
$$

[1] P. P. Kronberg, Rep. Prog. Phys. 57, 325 (1994).

[2] E. G. Zweibel and C. Heiles, Nature (London) 385, 131 (1997); K. T. Kim, P. P. Kronberg, and P.C. Tribble, Astrophys. J. 379, 80 (1991).

[3] Y.B. Zeldovich, A. A. Ruzmaikin, and D. D. Sokoloff, Magnetic Fields in Astrophysics (McGraw Hill, New York, 1980).

[4] L. M. Widrow, Rev. Mod. Phys. 74, 775 (2002).

[5] R. M. Athreya, V. K. Kapahi, P. J. McCarthy, and W. van Breugel, Astron. Astrophys. 329, 809 (1998).

[6] D. Grasso and H.R. Rubinstein, Phys. Rep. 348, 163 (2001); M. Giovannini, Int. J. Mod. Phys. D 13, 391 (2004).

[7] K. Jedamzik, V. Katalinic, and A. V. Olinto, Phys. Rev. D 57, 3264 (1998).

[8] K. Subramanian and J.D. Barrow, Phys. Rev. D 58, 083502 (1998).

[9] J. Silk, Astrophys. J. 151, 459 (1968).

[10] R. Banerjee and K. Jedamzik, Phys. Rev. D 70, 123003 (2004).

[11] R. Banerjee and K. Jedamzik, Phys. Rev. Lett. 91, 251301 (2003).

[12] B. Cheng, A. V. Olinto, D. N. Schramm, and J. W. Truran, Phys. Rev. D 54, 4714 (1996); P. J. Kernan, G. D.
Starkman, and T. Vachaspati, Phys. Rev. D 54, 7207 (1996).

[13] J. D. Barrow, P. G. Ferreira, and J. Silk, Phys. Rev. Lett. 78, 3610 (1997).

[14] J. Adams, U. H. Danielsson, D. Grasso, and H. Rubinstein, Phys. Lett. B 388, 253 (1996).

[15] K. Jedamzik, V. Katalinic, and A. V. Olinto, Phys. Rev. Lett. 85, 700 (2000).

[16] K. Subramanian and J.D. Barrow, Phys. Rev. Lett. 81, 3575 (1998); K. Subramanian and J. D. Barrow, Mon. Not. R. Astron. Soc. 335, L57 (2002).

[17] A. Mack, T. Kahniashvili, and A. Kosowsky, Phys. Rev. D 65, 123004 (2002).

[18] A. Lewis, Phys. Rev. D 70, 043011 (2004).

[19] D. G. Yamazaki, K. Ichiki, and T. Kajino, Astrophys. J., 625, L1 (2005).

[20] T. R. Seshadri and K. Subramanian, Phys. Rev. Lett., 87, 101301 (2001).

[21] U. Seljak and M. Zaldarriaga, Phys. Rev. Lett. 78, 2054 (1997); M. Zaldarriaga and U. Seljak, Phys. Rev. D 55, 1830 (1997); M. Kamionkowski, A. Kosowsky, and A. Stebbins, Phys. Rev. D, 55, 7368 (1997).

[22] K. Subramanian, T. R. Seshadri, and J. D. Barrow, Mon. Not. R. Astron. Soc. 344, L31 (2003). 


\section{HIROYUKI TASHIRO, NAOSHI SUGIYAMA, AND ROBI BANERJEE}

PHYSICAL REVIEW D 73, 023002 (2006)

[23] D. N. Spergel et al., Astrophys. J. Suppl. Ser. 148, 175 (2003).

[24] S. Weinberg, Astrophys. J. 168, 175 (1971).

[25] P. J. E. Peebles, Astrophys. J. 142, 1317 (1965).

[26] H. Kodama and M. Sasaki, Prog. Theor. Phys. Suppl. 78, 1 (1984).

[27] W. Hu and M. White, Phys. Rev. D 56, 596 (1997).

[28] A. Lewis, L. Challior, and A. Lasnby, Astrophys. J. 538, 473 (2000).

[29] C. L. Bennett et al., Astrophys. J. Suppl. Ser. 148, 1B (2003).
[30] W. C. Jones et al., astro-ph/0507494.

[31] T. J. Pearson et al., Astrophys. J. 591, 556 (2003).

[32] http://lambda.gsfc.nasa.gov/links/experimental_sites.cfm

[33] K. Dolagag, D. Grasso, V. Springel, and I. Tkachev, J. Cosmol. Astropart. Phys. 1 (2005) 9.

[34] http://www.auger.org/

[35] A. Letessier-Sevon, astro-ph/0510627.

[36] K. Greisen, Phys. Rev. Lett., 16, 748 (1966); G. T. Zatsepin, and V. A. Kuzmin, JETP Lett. 4, 78 (1966).

[37] G. Sigl, F. Miniati, and T. A. Enßlin, Phys. Rev. D 70, 043007 (2004). 